J. DIFFERENTIAL GEOMETRY

75 (2007) 303-358

\title{
A MATRIX LI-YAU-HAMILTON ESTIMATE FOR KÄHLER-RICCI FLOW
}

\author{
LEI NI
}

\begin{abstract}
In this paper we prove a new matrix Li-Yau-Hamilton ( $L Y H)$ estimate for Kähler-Ricci flow on manifolds with nonnegative bisectional curvature. The form of this new LYH estimate is obtained by the interpolation consideration originated in $[\mathbf{C h}]$ by Chow. This new inequality is shown to be connected with Perelman's entropy formula through a family of differential equalities. In the rest of the paper, we show several applications of this new estimate and its corresponding estimate for linear heat equation. These include a sharp heat kernel comparison theorem, generalizing the earlier result of $\mathrm{Li}$ and Tian $[\mathbf{L T}]$, a manifold version of Stoll's theorem [St] on the characterization of 'algebraic divisors', and a localized monotonicity formula for analytic subvarieties, which sharpens the Bishop volume comparison theorem.

Motivated by the connection between the heat kernel estimate and the reduced volume monotonicity of Perelman $[\mathbf{P}]$, we prove a sharp lower bound of the fundamental solution to the forward conjugate heat equation, which in a certain sense dual to Perelman's monotonicity of the reduced volume. As an application of this new monotonicity formula, we show that the blow-down limit of a certain type of long-time solution is a gradient expanding soliton, generalizing an earlier result of Cao. We also illustrate the connection between the new LYH estimate and the Hessian comparison theorem of [FIN] on the forward reduced distance. Localized monotonicity formulae on entropy and forward reduced volume are also derived.
\end{abstract}

\section{Introduction}

In $[\mathbf{L Y}]$, Peter Li and S.-T. Yau developed the fundamental gradient estimates for any positive solution $u(x, t)$ to the Schrödinger equation, which in particular applies to $u(x, t)$ satisfying the heat equation $\left(\frac{\partial}{\partial t}-\Delta\right) u(x, t)=0$. On a complete Riemannian manifold $M$ with Ricci curvature bounded from below, they also show that one can derive a

The author was supported in part by NSF Grants and an Alfred P. Sloan Fellowship, USA.

Received 10/09/2004. 
sharp form of the classical Harnack inequality (cf. [Mo]) out of their gradient estimates. The gradient estimate of Li-Yau is equivalent to a lower estimate on $\Delta \log u$. Later in [H2], Richard Hamilton extended the estimate of Li-Yau to the full matrix version of a sharp lower estimate on the Hessian of $\log u$, under the stronger assumption that $M$ is Ricci parallel and of nonnegative sectional curvature. More recently, in $[\mathbf{C N}]$, we observed that if $M$ is a Kähler manifold with nonnegative bisectional curvature, one can obtain a matrix version lower estimate on the complex Hessian of $\log u$ without the assumption of Ricci being parallel. Following $[\mathbf{N T 1}]$, in $[\mathbf{C N}]$ we called our estimate a LYH estimate (which is also referred to as a differential Harnack inequality in some earlier literatures). For Ricci flow there exists the fundamental work of Hamilton [H1] on the matrix LYH inequality for curvature tensors. For a similar result for Kähler-Ricci flow, please see [C1]. See also $[\mathbf{C C 1}, \mathbf{C C 2}],[\mathbf{C H}],[\mathbf{C K 1}],[\mathbf{N T 1}]$, etc, for the further developments related to LYH inequalities for Ricci/Kähler-Ricci flow, $[\mathbf{A}]$ and references therein for the LYH estimates for other geometric flows. The relation between $\mathrm{LYH}$ estimates and the monotonicity formulae was discussed in $[\mathbf{N 2}, \mathbf{N} 4]$.

In this paper we shall prove a new LYH estimate for time dependent Kähler metrics evolving by Kähler-Ricci flow. The new matrix inequality asserts that if $(M, g(t))$ is a complete solution to Kähler-Ricci flow $\frac{\partial}{\partial t} g_{\alpha \bar{\beta}}(x, t)=-R_{\alpha \bar{\beta}}(x, t)$ with (bounded, in case $M$ is non-compact) nonnegative bisectional curvature, and if $u$ is a positive solution to the forward conjugate heat equation: $\left(\frac{\partial}{\partial t}-\Delta-\mathcal{R}\right) u(x, t)=0$, where $\mathcal{R}$ is the scalar curvature, then

$$
u_{\alpha \bar{\beta}}+\frac{u}{t} g_{\alpha \bar{\beta}}+u R_{\alpha \bar{\beta}}+u_{\alpha} V_{\bar{\beta}}+u_{\bar{\beta}} V_{\alpha}+u V_{\alpha} V_{\bar{\beta}} \geq 0
$$

for any $(1,0)$ vector field $V$. Here $R_{\alpha \bar{\beta}}(x, t)$ is the Ricci tensor of $(M, g(t))$. We discover the above LYH estimate through an interpolation consideration originated in $[\mathbf{C h}]$. Below we shall illustrate this interpolation consideration further.

In $[\mathbf{C H}]$, Ben Chow and Richard Hamilton proved a linear trace LYH inequality for the symmetric positive definite 2-tensors evolved by the (time-dependent) Lichnerowicz heat equation, coupled with the Ricci flow, whose complete solution metrics have a bounded non-negative curvature operator. This result in particular generalizes the trace form of Hamilton's fundamental matrix LYH estimate [H1] on curvature tensors, for solutions to Ricci flow. Later, in $[\mathbf{C h}]$, Chow discovered an interesting interpolation phenomenon. Namely he shows a family of LYH estimates in the case of Riemann surfaces with positive curvature such that this family connects Li-Yau's estimate for the positive solutions to a heat equation, with Chow-Hamilton's linear trace estimate on the solutions to the Lichnerowicz heat equation (coupled with the Ricci flow) 
in the case of Riemann surfaces. An interesting question is whether or not this interpolation exists for high dimensional Ricci flow. Seeking the analogue of such interpolation in high dimension turns out to be fruitful. Even though the interpolation itself has not been found directly useful in geometric problems, it does play a crucial role in discovering new (useful) estimates. For example in [N4], such consideration led the author to discover a LYH estimate for the Hermitian-Einstein flow (what proved there is more general). This estimate of [N4] is one of the (crucial) new ingredients in obtaining the (sharp) estimates on the dimension of the spaces of holomorphic functions (sections of certain line bundles) of the polynomial growth. (For more details, please see [N4].) The LYH inequality of $[\mathbf{N} 4]$ can be interpolated with the earlier one discovered by L.-F. Tam and the author in [NT1]. Indeed, the interpolating estimate proved in Theorem 1.3 of $[\mathbf{N} 4]$ does provide a high dimensional generalization of $[\mathbf{C h}]$. Contemplation on this successful generalization further suggests that there may be a one-one correspondence, for the LYH estimates, between the linear case and the (nonlinear) case with Ricci flow. Seeking the linear correspondence of the LYH inequality for Kähler-Ricci flow in [NT1] leads to the correct formulation of the LYH's inequality in [N4] for the linear case. On the other hand, seeking for the nonlinear version of matrix LYH estimate of $[\mathbf{C N}]$ leads us to formulate the correct form of the (nonlinear version) matrix LYH inequality in this paper for the Kähler-Ricci flow. (Our naive 'one-one corresponding principle' was also reinforced by the similarity on the entropy formulae between the linear heat equation $[\mathbf{N 3}]$ and Ricci flow case $[\mathbf{P}]$.) The proof of this result is as usual through the tensor maximum principle of Hamilton [H2]. It could not have been completed without generous help from Ben Chow on a certain important step. We want to record our gratitude to him here.

The new estimate (1.1) is sharp since it holds equality if and only if on gradient expanding Kähler-Ricci solitons. Its proof also makes use of the earlier LYH estimate of [C1]. The Riemannian version of (1.1) suggests a new matrix differential inequality on curvature tensors, which is different from Hamilton's one in $[\mathbf{H 1}]$. Please see Section 6 for details. This new expression also vanishes identically on expanding solitons. Unfortunately, we have not been able to verify these new matrix estimates at this moment. (See Remark 6.4 for details.)

A little surprisingly, this new LYH estimate for the Kähler-Ricci flow can be shown related with Perelman's celebrated entropy formula for the Ricci flow $[\mathbf{P}]$, at least for Kähler case. Again this is done through the interpolation consideration explained above. Namely, one in fact can obtain a family of pre-LYH equalities (a notion suggested to us by Tom Ilmanen) such that at one end, the trace of this pre- $L Y H$ equality gives the Perelman's entropy formula after integrating on the manifold, 
and at the other end one obtains the LYH inequality of this paper by applying the tensor maximum principle of Hamilton. At the midpoint one obtains both the entropy formula of [N3] and the Li-Yau's estimate in $[\mathbf{L Y}]$ for the linear heat equation. (The pre- $L Y H$ equalities were proved earlier in [CLN] for the backward Ricci flow on Riemannian manifolds soon after the proof of (1.1).) This connection between (1.1) and Perelman's entropy formula suggests that the new LYH estimate proved here may be of some use or importance. Please see Section 4 for the detailed exposition on this interpolation between Perelman's entropy formula and the new LYH estimate. One should also refer to the recent beautiful survey [Ev1] and the stellar notes [Ev2] by Evans for the relation between entropy and the Harnack estimates for the linear heat equation (see also [N3] for a different connection). [Ev1] also contains other applications of entropy consideration in the study of PDE.

The rest of the paper is on applications of this new estimate, as well as the corresponding linear one of $[\mathbf{C N}]$. The immediate consequences include the monotonicity of a quantity called Nash's entropy, a Perelman type monotonicity (or Huisken type in the linear case) of the 'reduced volume of analytic subvarieties' and a sharp form of Harnack estimate for positive solutions to the forward conjugate heat equation. It also can be applied to proved a sharp heat kernel comparison theorem for any subvariety in complete Kähler manifolds with nonnegative bisectional curvature. This in a sense generalizes a previous result $[\mathbf{L T}]$, in which Peter $\mathrm{Li}$ and G. Tian proved the sharp comparison theorem on heat kernels of algebraic manifolds, equipped with the induced Fubini-Study metric (also called Bergmann metric in $[\mathbf{L T}]$ ) from the ambient $\mathbb{P}^{m}$. More precisely, we proved the following result.

Let $M$ be a complete Kähler manifold with nonnegative bisectional curvature. Let $H(x, y, t)$ be the fundamental solution of the heat equation. Let $\mathcal{V} \subset M$ be any complex subvariety of dimension s. Let $K_{\mathcal{V}}(x, y, t)$ be the fundamental solution of heat equation on $\mathcal{V}$. Then

$$
K_{\mathcal{V}}(x, y, t) \leq(\pi t)^{m-s} H(x, y, t), \text { for any } x, y \in \mathcal{V} .
$$

The equality implies that $\mathcal{V}$ is totally geodesic.

A slightly more involved application is a localized monotonicity formula and an elliptic 'monotonicity principle' for the analytic subvarieties. The latter leads to a manifold (curved/nonlinear) version of Stoll's characterization on 'algebraic divisors'. This localization uses, substantially, the beautiful ideas from the the study of mean curvature flow discovered by Ecker in $[\mathbf{E} 1]$ and $[\mathbf{E 2}]$. We give a brief sketch on these results below.

Let $\mathcal{V}$ be a subvariety of complex dimension $s$ as above. Denote by $\mathcal{A}_{\mathcal{V}, x_{0}}(\rho)$ the $2 s$-dimensional Hausdorff measure of $\mathcal{V} \cap B_{x_{0}}(\rho)$. Here 
$B_{x_{0}}(\rho)$ is the ball (inside $\left.M\right)$ of radius $\rho$ centered at $x_{0}$. The elliptic 'monotonicity principle' states the following:

There exists $C=C(m, s)$ such that for any $\rho^{\prime} \in(0, \delta(s) \rho)$

$$
\frac{\mathcal{A}_{\mathcal{V}, x_{0}}\left(\rho^{\prime}\right)\left(\rho^{\prime}\right)^{2(m-s)}}{V_{x_{0}}\left(\rho^{\prime}\right)} \leq C(m, s) \frac{\mathcal{A}_{\mathcal{V}, x_{0}}(\rho) \rho^{2(m-s)}}{V_{x_{0}}(\rho)} .
$$

Here $\delta(s)=\frac{1}{\sqrt{2+4 s}}$.

Notice that the above generalizes the monotonicity of [N4] from complex hyper-surfaces defined by a holomorphic function to complex subvarieties of arbitrary codimension. The following consequence of (1.3) is interesting to us.

Let $M^{m}$ be a complete Kähler manifold with nonnegative holomorphic bisectional curvature. Suppose that $M$ contains a compact subvariety $\mathcal{V}$ of complex dimension s. Then there exists $C=C(m, s)>0$ such that for $\delta(s) \rho \geq \rho^{\prime} \gg 1$

$$
\frac{V_{x_{0}}(\rho)}{V_{x_{0}}\left(\rho^{\prime}\right)} \leq C\left(\frac{\rho}{\rho^{\prime}}\right)^{2(m-s)}
$$

In particular,

$$
\limsup _{\rho \rightarrow \infty} \frac{V_{x_{0}}(\rho)}{\rho^{2(m-s)}}<\infty
$$

The result sharpens the Bishop-Gromov volume comparison theorem in the presence of compact subvarieties. When $M$ is simply-connected the result is in fact a consequence of the splitting theorem proved in Theorem 0.4 of [NT2], via a completely different approach. However, it is not clear that the result can be derived out of any previously known result in the general case when $M$ is not simply-connected. For an entire analytic set $\mathcal{V}$ in $\mathbb{C}^{m}$ (of dimension $s$ ), one can define the Lelong number at infinity (with respect to any fixed point $x_{0}$ ) by

$$
\nu_{\infty}\left(\mathcal{V}, x_{0}\right)=\limsup _{\rho \rightarrow \infty} \frac{\left(\pi \rho^{2}\right)^{(m-s)} \mathcal{A}_{\mathcal{V}, x_{0}}(\rho)}{V_{x_{0}}(\rho)} .
$$

Stoll showed that $\mathcal{V}$ is algebraic if and only if $\nu_{\infty}\left(\mathcal{V}, x_{0}\right)<\infty$ for some $x_{0}$. The following result generalizes his result to analytic sets in curved manifolds, for the codimension one case.

Let $M$ be a complete Kähler manifold with nonnegative bisectional curvature. Let $\mathcal{V}$ be an analytic divisor of $M$. Then $\mathcal{V}$ is defined by a 'polynomial function' (holomorphic function of polynomial growth) if and only if $\nu_{\infty}\left(\mathcal{V}, x_{0}\right)<\infty$, for some $x_{0} \in M$.

Besides the above results via the local monotonicity formula, Section 5 also contains some other results, one of which generalizes the classical transcendental Bézout estimate for codimension one analytic sets. (It 
has been known that the result is false for the high codimension case [CS], even for the manifold $M$ is the Euclidean spaces $\mathbb{C}^{m}$.) More precisely, we showed the following.

Let $M$ be a complete noncompact Kähler manifold with non-negative Ricci curvature. Let $f \in \mathcal{O}(M)$ be a holomorphic function of finite order. Let $Z(f)$ be the zero divisor of $f$. Then $\operatorname{Ord}\left(N_{Z}\left(x_{0}, r\right)\right) \leq$ $\operatorname{Ord}_{H}(f)$.

Here $N_{Z}\left(x_{0}, r\right)$ is the Nevanlinna counting function. Please see Section 5 for more details on the notations involved in the above result. We find the connection between the parabolic approach, especially the LYH estimate, and the classical Nevanlinna theory interesting and we believe that the parabolic approach should be the most natural/effective approach in extending sharp results from Euclidean spaces (linear) to the curved complex manifolds (nonlinear, in a certain sense).

Finally, we discuss the relationship between the LYH inequality proved in this paper and the previous computations in $[\mathbf{F I N}]$ on the reduced distance and the reduced volume modelled on Ricci expanders (in [FIN] we also call them forward reduced distance and forward reduced volume). In particular, we prove a sharp lower bound on the heat kernel for the time dependent metric evolving by Ricci flow, which, in a sense, is dual to Perelman's monotonicity of his reduced volume. We also illustrate how one can view this result, and more importantly, Perelman's monotonicity of the reduced volume, as a nonlinear analogue of earlier work of Cheeger-Yau $[\mathbf{C Y}]$ and Li-Yau $[\mathbf{L Y}]$ on the heat kernel estimates for the heat equation/Schrödinger equation. (Quite remarkably, Perelman's result requires no curvature sign assumption, while all the pervious results require either the nonnegativity of the Ricci or the Ricci curvature being bounded from below.) We also prove several localized monotonicity formulae for Ricci flow on the forward reduced volume of [FIN] and Perelman's entropy, without any curvature sign assumptions. This again follows the ideas of Ecker in [E2], where a localized version of Huisken's monotonicity formula is derived for the mean curvature flow. As an application of the new monotonicity formula on the second forward reduced volume defined in this paper (which is slightly different from the forward reduced volume of [FIN], but formally is the same as the reduced volume of $[\mathbf{P}]$ ), we prove that the blow-down limit, with respect to any sequence of space-time points $\left(x_{k}, t_{k}\right)$ with $t_{k} \rightarrow \infty$ and $\frac{r_{0}^{2}\left(x_{k}, x_{0}\right)}{t_{k}} \leq C$, for some fixed point $x_{0} \in M$ and $C>0$ (where $r_{0}(x, y)$ is the distance function with respect to the initial metric $g(0)$ ), of a so-called type III $\kappa$-solution to Kähler-Ricci flow with bounded nonnegative bisectional curvature must be a gradient expanding soliton. This is, in a certain sense, dual to Perelman's result in Section 11 of $[\mathbf{P}]$ on the blow-down limit of certain type of ancient solutions. This result was proved for the 
sequence of specially chosen space-time points (maximum points of the scalar curvature) earlier in [C2] and for the space time sequence of the special form $\left(x_{0}, t_{k}\right)$ in [CT2] very recently (independently). Note that our approach works for both Ricci and Kähler-Ricci flow.

Here is how we organize this paper. In Section 2 we prove the interpolating version of the new LYH estimate, which in particular includes (1.1). In Section 3 we derive some monotonicity formulae and the heat kernel comparison theorems out of this new estimate. In Section 4 we show the interpolation between the new LYH inequality of this paper and Perelman's entropy formula in $[\mathbf{P}]$. In Section 5 we derive the localized monotonicity formula and prove the manifold version of Stoll's theorem. In Section 6, we discuss the relation between the new inequality and the work of [FIN], which motivates us to formulate a new matrix LYH expression on curvature tensors of metrics evolving by Ricci flow, and to show a sharp heat kernel lower bound estimate. In Section 6 , we also derive several localized monotonicity formulae, including two entropy monotonicity formulae localizing the Perelman's celebrated entropy formula in $[\mathbf{P}]$.

Acknowledgement. The special thanks go to B. Chow since his contribution is crucial to some results in this paper. He generously encouraged the author to publish the results alone even though it should really be a joint paper. The author would also like to thank H.-D. Cao, T. Ilmanen, P. Li and J. Wang for helpful discussions; K. Ecker, L.-F. Tam, H. Wu for their interests.

\section{A new matrix LYH inequality for Kähler-Ricci flow}

Let $M^{m}$ be a complete Kähler manifold of complex dimension $m$. Let $(M, g(t))$ be a solution to Kähler-Ricci flow:

$$
\frac{\partial}{\partial t} g_{\alpha \bar{\beta}}(x, t)=-R_{\alpha \bar{\beta}}(x, t) .
$$

Here $R_{\alpha \bar{\beta}}(x, t)$ is the Ricci tensor of the metric $g_{\alpha \bar{\beta}}(x, t)$. Let $u$ be a positive solution to the forward conjugate heat equation:

$$
\left(\frac{\partial}{\partial t}-\Delta\right) u(x, t)=\mathcal{R}(x, t) u(x, t) .
$$

Here $\mathcal{R}(x, t)$ is the scalar curvature. We shall prove the following new matrix LYH/differential Harnack inequality.

Theorem 2.1. Let $(M, g(t))$ be a solution defined on $M \times[0, T]$ (for some $T>0)$ to $(2.1)$ with nonnegative bisectional curvature. In the case that $M$ is complete noncompact, assume further that the bisectional curvature is bounded on $M \times[0, T]$. Let $u$ be a positive solution to (2.2). Then

$$
u_{\alpha \bar{\beta}}+\frac{u}{t} g_{\alpha \bar{\beta}}+u R_{\alpha \bar{\beta}}+u_{\alpha} V_{\bar{\beta}}+u_{\bar{\beta}} V_{\alpha}+u V_{\alpha} V_{\bar{\beta}} \geq 0
$$


for any $(1,0)$ vector field $V$.

The assumption that $(M, g(t))$ has bounded nonnegative bisectional curvature can be replaced by one that $(M, g(0))$ has nonnegative bisectional curvature and $(M, g(t))$ has bounded curvature, thanks to the result of $[\mathbf{B}],[\mathbf{M} 2]$ and $[\mathbf{S h 2}]$. The same applies to other results of this paper. We state the result under the stronger assumption just for simplicity.

Recall that in $[\mathbf{C N}]$ the authors proved that on a Kähler manifold $\left(M, g_{\alpha \bar{\beta}}(x)\right)$ with the nonnegative bisectional curvature, for any positive solution $u$ to the heat equation $\left(\frac{\partial}{\partial t}-\Delta\right) u=0$, one has the matrix LYH inequality:

$$
u_{\alpha \bar{\beta}}+\frac{u}{t} g_{\alpha \bar{\beta}}+u_{\alpha} V_{\bar{\beta}}+u_{\bar{\beta}} V_{\alpha}+u V_{\alpha} V_{\bar{\beta}} \geq 0 .
$$

Hence one can think (2.3) is the nonlinear version of (2.4). Moreover, we shall show that there exists a linear interpolation between these two inequalities. The similar interpolation was established in $[\mathbf{C h}]$ originally for the Li-Yau's gradient estimates for the heat equation and Hamilton's differential Harnack for the Ricci flow in the case of surfaces. We shall show such interpolation between the matrix differential inequalities (2.3) and (2.4) for any dimensions. In [N4] we have shown another family of LYH inequalities which also serves as a high dimensional generalization of $[\mathbf{C h}]$.

Let us first set up the notations. For any $\epsilon>0$, we consider the Kähler-Ricci flow:

$$
\frac{\partial}{\partial t} g_{\alpha \bar{\beta}}(x, t)=-\epsilon R_{\alpha \bar{\beta}}(x, t) .
$$

Consider the positive solution $u$ to the parabolic equation:

$$
\left(\frac{\partial}{\partial t}-\Delta\right) u(x, t)=\epsilon \mathcal{R}(x, t) u(x, t) .
$$

We shall call (2.6) forward conjugate heat equation, which is the adjoint of the backward heat equation $\left(\frac{\partial}{\partial t}+\Delta\right) v=0$. We shall prove the following interpolation theorem.

Theorem 2.2 (Chow-Ni). Assume that the complete solution $(M, g(t))$ (defined on $M \times[0, T]$ for some $T>0)$ to (2.5) has nonnegative bisectional curvature. In the case that $M$ is noncompact, assume further that the bisectional curvature of $g(t)$ is uniformly bounded on $M \times[0, T]$. Let $u$ be a positive solution to (2.6). Then

$$
u_{\alpha \bar{\beta}}+\frac{u}{t} g_{\alpha \bar{\beta}}+\epsilon u R_{\alpha \bar{\beta}}+u_{\alpha} V_{\bar{\beta}}+u_{\bar{\beta}} V_{\alpha}+u V_{\alpha} V_{\bar{\beta}} \geq 0
$$

for any $(1,0)$ vector field $V$. 
It is easy to see that Theorem 2.2, serving an interpolation between (2.3) and (2.4), implies Theorem 2.1 and the earlier result (2.4) for the linear heat equation. Therefore, we only need to prove Theorem 2.2. The proof consists of the following several lemmas.

\section{Lemma 2.3.}

$$
(\mathcal{R})_{\alpha \bar{\beta}}=\Delta R_{\alpha \bar{\beta}}+R_{\alpha \bar{\beta} \gamma \bar{\delta}} R_{\bar{\delta} \gamma}-R_{\alpha \bar{p}} R_{p \bar{\beta}}
$$

Here $R_{\alpha \bar{\beta} \gamma \bar{\delta}}$ is curvature tensor in terms of holomorphic coordinates.

Proof. The second Bianchi identity yields

$$
\begin{aligned}
\mathcal{R}_{\alpha \bar{\beta}} & =\left(R_{\gamma \bar{\gamma}}\right)_{, \alpha \bar{\beta}}=R_{\alpha \bar{\gamma}, \gamma \bar{\beta}} \\
& =R_{\alpha \bar{\gamma}, \bar{\beta} \gamma}-R_{p \bar{\gamma} \gamma \bar{\beta}} R_{\alpha \bar{p}}+R_{\alpha \bar{p} \gamma \bar{\beta}} R_{p \bar{\gamma}} \\
& =R_{\alpha \bar{\beta}, \bar{\gamma} \gamma}-R_{p \bar{\beta}} R_{\alpha \bar{p}}+R_{\alpha \bar{p} \gamma \bar{\beta}} R_{p \bar{\gamma}} .
\end{aligned}
$$

The commutator formula gives that

$$
\begin{aligned}
R_{\alpha \bar{\beta}, \bar{\gamma} \gamma} & =R_{\alpha \bar{\beta}, \gamma \bar{\gamma}}-R_{p \bar{\beta} \gamma \bar{\gamma}} R_{\alpha \bar{p}}+R_{\alpha \bar{p} \gamma \bar{\gamma}} R_{p \bar{\beta}} \\
& =R_{\alpha \bar{\beta}, \gamma \bar{\gamma}}-R_{p \bar{\beta}} R_{\alpha \bar{p}}+R_{\alpha \bar{p}} R_{p \bar{\beta}} .
\end{aligned}
$$

The lemma now follows from the definition $\Delta R_{\alpha \bar{\beta}}=\frac{1}{2}\left(R_{\alpha \bar{\beta}, \gamma \bar{\gamma}}+R_{\alpha \bar{\beta}, \bar{\gamma} \gamma}\right)$. q.e.d.

Lemma 2.4. Let $u(x, t)$ be a solution to (2.6). Then

$$
\left(\frac{\partial}{\partial t}-\Delta\right) u_{\alpha \bar{\beta}}=R_{\alpha \bar{\beta} \gamma \bar{\delta}} u_{\bar{\gamma} \delta}-\frac{1}{2}\left(R_{\alpha \bar{p}} u_{p \bar{\beta}}+R_{p \bar{\beta}} u_{\alpha \bar{p}}\right)+\epsilon(\mathcal{R} u)_{\alpha \bar{\beta}} .
$$

Proof. Differentiate (2.6) we have

$$
\left(u_{t}\right)_{\gamma \bar{\delta}}=R_{\beta \bar{\alpha} \gamma \bar{\delta}} u_{\alpha \bar{\beta}}+g^{\alpha \bar{\beta}} u_{\alpha \bar{\beta} \gamma \bar{\delta}}+\epsilon(\mathcal{R} u)_{\gamma \bar{\delta}} .
$$

By definition $\Delta u_{\alpha \bar{\beta}}=\frac{1}{2}\left(u_{\alpha \bar{\beta}, \gamma \bar{\gamma}}+u_{\alpha \bar{\beta}, \bar{\gamma} \gamma}\right)$, with respect to a normal coordinate centered at any fixed point. We need to calculate the difference between the partial derivative $u_{\alpha \bar{\beta} \gamma \bar{\delta}}$ and the covariant derivative $u_{\alpha \bar{\beta}, \gamma \bar{\delta}}$. Direct computations show that, in a normal coordinate centered at any fixed point,

$$
u_{\gamma \bar{\delta}, \alpha \bar{\beta}}=u_{\gamma \bar{\delta} \alpha \bar{\beta}}+u_{s \bar{\delta}} R_{\alpha \bar{\beta} \gamma \bar{s}} .
$$

Using the fact that

$$
u_{\gamma \bar{\delta}, \alpha \bar{\alpha}}=u_{\gamma \bar{\delta}, \bar{\alpha} \alpha}+R_{\gamma \bar{p}} u_{p \bar{\delta}}-R_{p \bar{\delta}} u_{\gamma \bar{p}}
$$

and (2.13) we have

$$
\begin{aligned}
\Delta u_{\gamma \bar{\delta}} & =\frac{1}{2}\left(u_{\gamma \bar{\delta}, \alpha \bar{\alpha}}+u_{\gamma \bar{\delta}, \bar{\alpha} \alpha}\right) \\
& =u_{\gamma \bar{\delta} \alpha \bar{\alpha}}+\frac{1}{2}\left(R_{\gamma \bar{p}} u_{p \bar{\delta}}+R_{p \bar{\delta}} u_{\gamma \bar{p}}\right) .
\end{aligned}
$$

Combining the above with (2.12), we conclude that $u_{\alpha \bar{\beta}}$ satisfies (2.11). 
The direct calculations give the following lemma.

Lemma 2.5. Let $(M, g(t))$ be a solution to $(2.5)$. Let $u(x, t)$ be a positive solution to (2.6). Then

$$
\begin{aligned}
& \left(\frac{\partial}{\partial t}-\Delta\right)\left(\frac{u_{\alpha} u_{\bar{\beta}}}{u}\right) \\
& =\epsilon \frac{(\mathcal{R} u)_{\alpha} u_{\bar{\beta}}}{u}+\epsilon \frac{(\mathcal{R} u)_{\bar{\beta}} u_{\alpha}}{u}-\epsilon(\mathcal{R} u) \frac{u_{\alpha} u_{\bar{\beta}}}{u^{2}} \\
& \quad-\frac{u_{\alpha \bar{s}} u_{\bar{\beta} s}+u_{\alpha s} u_{\bar{\beta} \bar{s}}}{u} \\
& \quad-2 \frac{u_{\alpha} u_{\bar{\beta}}\left|u_{s}\right|^{2}}{u}-\frac{1}{2} \frac{R_{\alpha \bar{s}} u_{s} u_{\bar{\beta}}+R_{s \bar{\beta}} u_{\bar{s}} u_{\alpha}}{u} \\
& \quad+\frac{u_{\alpha s} u_{\bar{s}} u_{\bar{\beta}}+u_{\bar{\beta} s} u_{\alpha} u_{\bar{s}}+u_{\alpha \bar{s}} u_{\bar{\beta}} u_{s}+u_{\bar{\beta} \bar{s}} u_{\alpha} u_{s}}{u^{2}} . \\
& \left(\frac{\partial}{\partial t}-\Delta\right)\left(\frac{u}{t} g_{\alpha \bar{\beta}}\right)(x, t)=\epsilon \frac{\mathcal{R} u}{t} g_{\alpha \bar{\beta}}-\frac{u}{t^{2}} g_{\alpha \bar{\beta}}-\epsilon \frac{u}{t} R_{\alpha \bar{\beta}} . \\
& \left(\frac{\partial}{\partial t}-\Delta\right)\left(\epsilon u R_{\alpha \bar{\beta}}\right) \\
& =\epsilon^{2} \mathcal{R} u R_{\alpha \bar{\beta}}+\epsilon u\left(R_{\alpha \bar{\beta} \gamma \bar{\delta}} R_{\bar{\gamma} \delta}-R_{\alpha \bar{p}} R_{p \bar{\beta}}\right) \\
& \quad \cdot \epsilon\left(\nabla_{s} u \nabla_{\bar{s}} R_{\alpha \bar{\beta}}+\nabla_{\bar{s}} u \nabla_{s} R_{\alpha \bar{\beta}}\right) \\
& \quad+\left(\epsilon^{2}-\epsilon\right) u\left(R_{\alpha \bar{\beta} \gamma \bar{\delta}} R_{\bar{\gamma} \delta}-R_{\alpha \bar{p}} R_{p \bar{\beta}}+\Delta R_{\alpha \bar{\beta}}\right) .
\end{aligned}
$$

Proof. The proof is straightforward computation. In the derivation of (2.16), a commutator formula has been used. In the derivation of (2.18) we have used

$$
\frac{\partial}{\partial t} R_{\alpha \bar{\beta}}=\epsilon\left(R_{\alpha \bar{\beta} \gamma \bar{\delta}} R_{\bar{\gamma} \delta}-R_{\alpha \bar{p}} R_{p \bar{\beta}}+\Delta R_{\alpha \bar{\beta}}\right) .
$$

q.e.d.

We also need the following result, which is an easy consequence of Cao's differential Harnack inequality for the Kähler-Ricci flow [C1].

Lemma 2.6. Let $(M, g(t))$ be a complete solution to (2.5) with nonnegative bisectional curvature. In the case that $M$ is noncompact we further assume that the bisectional curvature is bounded. Let $u(x, t)$ be a positive solution to (2.6). Then

$$
\begin{aligned}
& \tilde{Y}_{\alpha \bar{\beta}}:=\Delta R_{\alpha \bar{\beta}}+R_{\alpha \bar{\beta} \gamma \bar{\delta}} R_{\bar{\gamma} \delta}-\left(\frac{\nabla_{s} u}{\epsilon u} \nabla_{\bar{s}} R_{\alpha \bar{\beta}}+\frac{\nabla_{\bar{s}} u}{\epsilon u} \nabla_{s} R_{\alpha \bar{\beta}}\right) \\
&+R_{\alpha \bar{\beta} \gamma \bar{\delta}} \frac{\nabla_{\bar{\gamma}} u}{\epsilon u} \frac{\nabla_{\delta} u}{\epsilon u}+\frac{R_{\alpha \bar{\beta}}}{\epsilon t} \geq 0 .
\end{aligned}
$$

Once we have Lemma 2.3-2.6 we can give the proof of Theorem 2.2. 
Proof of Theorem 2.2. Let

$$
N_{\alpha \bar{\beta}}=u_{\alpha \bar{\beta}}+\frac{u}{t} g_{\alpha \bar{\beta}}-\frac{u_{\alpha} u_{\bar{\beta}}}{u}
$$

and let

$$
\tilde{N}_{\alpha \bar{\beta}}=N_{\alpha \bar{\beta}}+\epsilon u R_{\alpha \bar{\beta}} .
$$

By taking the minimizing vector in (2.7), one can see that the claimed inequality in Theorem 2.2 is equivalent to the assertion that $\tilde{N}_{\alpha \bar{\beta}} \geq$ 0 . When $M$ is noncompact, the maximum principle (either for scalar functions or for tensors) is false in general. It only holds under certain growth conditions for the noncompact manifolds. We shall first prove the theorem for the relatively easier case when $M$ is compact.

Compact case. By Lemma 2.3-2.5, we have that

$$
\begin{aligned}
& \left(\frac{\partial}{\partial t}-\Delta\right) \tilde{N}_{\alpha \bar{\beta}} \\
& =R_{\alpha \bar{\beta} \gamma \bar{\delta}} u_{\bar{\gamma} \delta}-\frac{1}{2}\left(R_{\alpha \bar{p}} u_{p \bar{\beta}}+R_{p \bar{\beta}} u_{\alpha \bar{p}}\right)+\epsilon(\mathcal{R} u)_{\alpha \bar{\beta}} \\
& \quad-\epsilon \frac{(\mathcal{R} u)_{\alpha} u_{\bar{\beta}}}{u}-\epsilon \frac{(\mathcal{R} u)_{\bar{\beta}} u_{\alpha}}{u}+\epsilon(\mathcal{R} u) \frac{u_{\alpha} u_{\bar{\beta}}}{u^{2}} \\
& \quad+\frac{u_{\alpha \bar{s}} u_{\bar{\beta} s}+u_{\alpha s} u_{\bar{\beta} \bar{s}}}{u} \\
& +2 \frac{u_{\alpha} u_{\bar{\beta}}\left|u_{s}\right|^{2}}{u}+\frac{1}{2} \frac{R_{\alpha \bar{s}} u_{s} u_{\bar{\beta}}+R_{s \bar{\beta}} u_{\bar{s}} u_{\alpha}}{u} \\
& \quad-\frac{u_{\alpha s} u_{\bar{s}} u_{\bar{\beta}}+u_{\bar{\beta} s} u_{\alpha} u_{\bar{s}}+u_{\alpha \bar{s}} u_{\bar{\beta}} u_{s}+u_{\bar{\beta} \bar{s}} u_{\alpha} u_{s}}{u^{2}} \\
& \quad+\epsilon \frac{\mathcal{R} u}{t} g_{\alpha \bar{\beta}}-\frac{u}{t^{2}} g_{\alpha \bar{\beta}}-\epsilon \frac{u}{t} R_{\alpha \bar{\beta}} \\
& +\epsilon^{2} \mathcal{R} u R_{\alpha \bar{\beta}}+\epsilon u\left(R_{\alpha \bar{\beta} \gamma \bar{\delta}} R_{\bar{\gamma} \delta}-R_{\alpha \bar{p}} R_{p \bar{\beta}}\right) \\
& \quad-\epsilon\left(\nabla_{s} u \nabla_{\bar{s}} R_{\alpha \bar{\beta}}+\nabla_{\bar{s}} u \nabla_{s} R_{\alpha \bar{\beta}}\right) \\
& \quad+\left(\epsilon^{2}-\epsilon\right) u\left(R_{\alpha \bar{\beta} \gamma \bar{\delta}} R_{\bar{\gamma} \delta}-R_{\alpha \bar{p}} R_{p \bar{\beta}}+\Delta R_{\alpha \bar{\beta}}\right) .
\end{aligned}
$$

Regrouping terms yields

$$
\begin{aligned}
& \left(\frac{\partial}{\partial t}-\Delta\right) \tilde{N}_{\alpha \bar{\beta}} \\
& =R_{\alpha \bar{\beta} \gamma \bar{\delta}} \tilde{N}_{\bar{\gamma} \delta}-\frac{1}{2}\left(R_{\alpha \bar{p}} \tilde{N}_{p \bar{\beta}}+R_{p \bar{\beta}} \tilde{N}_{\alpha \bar{p}}\right)-\frac{2}{t} \tilde{N}_{\alpha \bar{\beta}} \\
& \quad+\frac{1}{u} N_{\alpha \bar{p}} N_{p \bar{\beta}}+\frac{1}{u}\left(u_{\alpha p}-\frac{u_{\alpha} u_{p}}{u}\right)\left(u_{\bar{p} \bar{\beta}}-\frac{u_{\bar{p}} u_{\bar{\beta}}}{u}\right) \\
& \quad+\epsilon \mathcal{R} \tilde{N}_{\alpha \bar{\beta}}-u \epsilon^{2} R_{\alpha \bar{p}} R_{p \bar{\beta}}+\epsilon^{2} u \tilde{Y}_{\alpha \bar{\beta}}
\end{aligned}
$$


where $\tilde{Y}_{\alpha \bar{\beta}}$ is the tensor defined in Lemma (2.6). Since Lemma 2.6 implies that $\tilde{Y}_{\alpha \bar{\beta}} \geq 0,(2.20)$ then implies that

$$
\begin{aligned}
& \left(\frac{\partial}{\partial t}-\Delta\right) \tilde{N}_{\alpha \bar{\beta}} \\
& =R_{\alpha \bar{\beta} \gamma \bar{\delta}} \tilde{N}_{\bar{\gamma} \delta}-\frac{1}{2}\left(R_{\alpha \bar{p}} \tilde{N}_{p \bar{\beta}}+R_{p \bar{\beta}} \tilde{N}_{\alpha \bar{p}}\right) \\
& \quad+\frac{1}{2 u} \tilde{N}_{\alpha \bar{p}}\left(N_{p \bar{\beta}}-\epsilon u R_{p \bar{\beta}}\right)+\frac{1}{2 u}\left(N_{\alpha \bar{p}}-\epsilon u R_{\alpha \bar{p}}\right) \tilde{N}_{p \bar{\beta}} \\
& \quad+\epsilon^{2} u \tilde{Y}_{\alpha \bar{\beta}}+\frac{1}{u}\left(u_{\alpha p}-\frac{u_{\alpha} u_{p}}{u}\right)\left(u_{\bar{p} \bar{\beta}}-\frac{u_{\bar{p}} u_{\bar{\beta}}}{u}\right) \\
& \quad+\left(\epsilon \mathcal{R}-\frac{2}{t}\right) \tilde{N}_{\alpha \bar{\beta}} \\
& \geq R_{\alpha \bar{\beta} \gamma \bar{\delta}} \tilde{N}_{\bar{\gamma} \delta}-\frac{1}{2}\left(R_{\alpha \bar{p}} \tilde{N}_{p \bar{\beta}}+R_{p \bar{\beta}} \tilde{N}_{\alpha \bar{p}}\right) \\
& \quad+\frac{1}{2 u} \tilde{N}_{\alpha \bar{p}}\left(N_{p \bar{\beta}}-\epsilon u R_{p \bar{\beta}}\right)+\frac{1}{2 u}\left(N_{\alpha \bar{p}}-\epsilon u R_{\alpha \bar{p}}\right) \tilde{N}_{p \bar{\beta}} \\
& \quad+\left(\epsilon \mathcal{R}-\frac{2}{t}\right) \tilde{N}_{\alpha \bar{\beta}} .
\end{aligned}
$$

Using the observation that the right hand side of (2.21) satisfies the nullvector condition of the tensor maximum principle of Hamilton [H2], we have proved the case with $M$ being compact.

Noncompact case. We have to evoke the tensor maximum principle of [N5]. Some integral estimates on up to second derivatives of $u$ are needed. First recall the fundamental derivative estimate of Shi. For $g_{\alpha \bar{\beta}}(x, t)$, a solution to (2.5) on $M \times[0, T]$ with bounded (in space-time) nonnegative bisectional curvature, there exist $A_{k}>0$ such that

$$
\left\|\nabla^{k} R_{\alpha \bar{\beta} \gamma \bar{\delta}}\right\|^{2} \leq \frac{A_{k}}{t^{k}}
$$

on $M \times[0, T]$. The estimate $(2.22)$ is proved in [Sh1]. For the sake of simplicity, we will show the matrix differential Harnack inequality Theorem 2.2 for the case $\epsilon=1$ under the estimates (2.22). In fact, what we need is $(2.22)$ for $k \leq 2$. It is clear that we only need to prove the theorem for sufficient small $T$ since one can iterate the process. For the proof we also need the perturbation trick from [NT1] (see also $[\mathbf{C N}]$ ). Namely we first shift $t$ by $2 \delta$, where $\delta$ is a small positive number. After the shifting we can have estimates on $u,|\nabla u|$ and $\left|u_{\alpha \bar{\beta}}\right|$. The goal is to show that there exists a constant $b>0$ such that

$$
\int_{\delta}^{T} \int_{M} \exp \left(-b\left(r_{0}^{2}(x)+1\right)\right)\left(\frac{1}{u}+\frac{|\nabla u|^{2}}{u}+\left|u_{\alpha \bar{\beta}}\right|^{2}\right) d \mu d t<\infty .
$$

Here $r_{0}(x)=r_{0}(o, x)$ is the distance to $x$ from a fixed point $o \in M$ with respect to the initial metric. We need the estimate (2.23) to apply the 
tensor maximum principle from [N5] (see also [NT2] for the original time-independent version).

In order to get control on $u$ (or $\frac{1}{u}$ ) first we need the following Harnack inequality of Guenther $[\mathbf{G u}]$.

Theorem 2.7 (Guenther). Let $(M, g(t))$ be a solution to Ricci flow satisfying (2.22). Let $u$ be a positive solution to the forward conjugate heat equation $\left(\frac{\partial}{\partial t}-\Delta\right) u=\mathcal{R} u$. Then for sufficiently small $T$ (only depending on $A_{k}$ ) there exist $\alpha, B_{1}>0$ only depending on $A_{k}$ such that

$$
u\left(x_{1}, t_{1}\right) \leq u\left(x_{2}, t_{2}\right)\left(\frac{t_{2}}{t_{1}}\right)^{\frac{n}{2}} \exp \left(\frac{r^{2}\left(x_{1}, x_{2}, t_{1}\right)}{\alpha\left(t_{2}-t_{1}\right)}+B_{1}\left(t_{2}-t_{1}\right)\right)
$$

for any $T \geq t_{2}>t_{1}>0$. Here $r\left(x_{1}, x_{2}, t_{1}\right)$ denotes the the distance between $x_{1}$ and $x_{2}$ with respect to the metric at $t=t_{1}$.

The result above was proved in $[\mathbf{G u}]$ through a gradient estimate of Li-Yau type on compact manifold. Since one can apply the localization techniques as on page 161 of [LY] (see also page 647 of [NT1], [Sh1] ) one can easily generalize the gradient estimate of $[\mathbf{G u}]$, thus the above Harnack estimate to complete noncompact case. From (2.24), one can deduce that for small $\delta$ there exists a constant $b_{2}, B_{2}>0$, where $b_{2}=$ $b_{2}\left(A_{k}, \delta\right)$ and $B_{2}=B_{2}\left(u\left(o, \frac{\delta}{4}\right), u\left(o, T-\frac{\delta}{4}\right), A_{k}, \delta\right)$ such that

$$
\left(\frac{1}{u}+u\right)(x, t) \leq B_{2} \exp \left(b_{2}\left(r_{0}^{2}(x)+1\right)\right)
$$

for $(x, t) \in M \times\left[\frac{\delta}{2}, T-\frac{\delta}{2}\right]$.

Observe that we have the following two equations.

$$
\left(\frac{\partial}{\partial t}-\Delta\right) u^{2}=\mathcal{R} u^{2}-|\nabla u|^{2}
$$

and

$$
\left(\frac{\partial}{\partial t}-\Delta\right)|\nabla u|^{2}=-\left|u_{\alpha \bar{\beta}}\right|^{2}-\left|u_{\alpha \beta}\right|^{2}+\langle\nabla(\mathcal{R} u), \nabla u\rangle+\langle\nabla u, \nabla(\mathcal{R} u)\rangle .
$$

The desired estimate (2.23) follows from (2.26), (2.27) by the argument through integration by parts as in Lemma 3.1 of $[\mathbf{C N}]$. Once we have established the estimate (2.23) we can apply the perturbation argument as in $[\mathbf{N T 1}]$ (see also $[\mathbf{C N}]$ ) together with the tensor maximum principle in Theorem 2.1 of [NT2] (see also Theorem 2.1 in [N5] for the Ricci flow case) to conclude the proof of the matrix LYH estimate (2.7) for the complete noncompact case. Note that Theorem 2.7 only provides the estimate for short time. But we can iterate the argument to prove the result for all time. An alternative is that once one has the upper bound estimate at some earlier time one can also make use of the heat kernel estimate in [N5] for the time dependent heat operator (and uniqueness 
of the positive solution) to get estimates of the positive solution for the later time. We also should remark that the matrix LYH (2.7) gives a sharp Harnack (which is more precise, compared with Theorem 2.7). Please see Corollary 3.8 in the next section. However, we do need the rough estimate in the proof to apply the tensor maximum principle. q.e.d.

Corollary 2.8. Let $u(x, t)$ be a positive solution to (2.6). Then

$$
\Delta \log u+\epsilon \mathcal{R}+\frac{m}{t} \geq 0 .
$$

If the equality holds for some $\left(x_{0}, t_{0}\right)$ with $t_{0}>0$, then $(M, g(t))$ is an expanding Kähler-Ricci soliton, for the case $\epsilon>0$, and $(M, g)$ is isometric to $\mathbb{C}^{m}$, for the case $\epsilon=0$.

Proof. Let

$$
Q=\Delta \log u+\epsilon \mathcal{R}+\frac{m}{t}
$$

and $\mathcal{Q}=g^{\alpha \bar{\beta}} \tilde{Y}_{\alpha \bar{\beta}}$. Since $\tilde{N}_{\alpha \bar{\beta}} \geq 0$ and $Q=g^{\alpha \bar{\beta}} \tilde{N}_{\alpha \bar{\beta}}$ we have that $\tilde{N}_{\alpha \bar{\beta}}\left(x_{0}, t_{0}\right)=0$. By the strong maximum principle we know that $\tilde{N}_{\alpha \bar{\beta}} \equiv$ 0 for all $t<t_{0} . \tilde{N}_{\alpha \bar{\beta}} \equiv 0$ is nothing but the equation in the definition of the gradient expanding soliton.

For the case $\epsilon=0$, we apply the same line of argument. In this case we have $Q \equiv 0$ since $\tilde{N}_{\alpha \bar{\beta}}=N_{\alpha \bar{\beta}} \equiv 0$, for $t \leq t_{0}$. Now from the equation

$$
\begin{aligned}
0= & \left(\frac{\partial}{\partial t}-\Delta\right) t^{2} Q \\
= & t^{2} R_{\alpha \bar{\beta}}(\log u)_{\bar{\alpha}}(\log u)_{\beta}+\frac{t^{2}}{u}\left(u_{\alpha p}-\frac{u_{\alpha} u_{p}}{u}\right)\left(u_{\bar{p} \bar{\alpha}}-\frac{u_{\bar{p}} u_{\bar{\alpha}}}{u}\right) \\
& +\frac{t^{2}}{u} N_{\alpha \bar{p}} N_{p \bar{\alpha}} \geq 0
\end{aligned}
$$

we have that $(\log u)_{\alpha \beta} \equiv 0$ too. From the definition of $N_{\alpha \bar{\beta}}$ we then have

$$
(\log u)_{\alpha \bar{\beta}}=\frac{1}{t} g_{\alpha \bar{\beta}}
$$

and

$$
(\log u)_{\alpha \beta}=0,
$$

from which it is easy to see that $(M, g)$ is flat and in fact isometric to $\mathbb{C}^{m}$, since the curvature (see, for example, page 117 of $[\mathbf{K M}]$ ) can be written as

$$
R_{\alpha \bar{\beta} \gamma \bar{\delta}}=-\frac{\partial^{4}(t f)}{\partial z^{\alpha} \partial z^{\bar{\beta}} \partial z^{\gamma} \partial z^{\bar{\delta}}}+g^{p \bar{q}}\left(\frac{\partial^{3}(t f)}{\partial z^{\bar{q}} \partial z^{\alpha} \partial z^{\gamma}}\right)\left(\frac{\partial^{3}(t f)}{\partial z^{p} \partial z^{\bar{\beta}} \partial z^{\bar{\delta}}}\right)
$$

and $\log u$ is a convex function.

q.e.d. 
Remark 2.9. The $\epsilon=0$ case in the above Corollary 2.8 is just the original Li-Yau's estimate $[\mathbf{L Y}]$, which holds with nonnegativity of the Ricci curvature. The assertion that the equality implies the manifold is isometric to $\mathbb{R}^{n}$ is implicit in the proofs of $[\mathbf{L Y}]$ and is proved explicitly in [N3] for Riemannian manifolds with nonnegative Ricci curvature. It would be interesting to see if Corollary 2.8 is true, for $\epsilon>0$, on any complete solution to the Kähler-Ricci/Ricci flow without assumptions on the sign of the curvature.

When the manifold is compact, we known that, by passing to its universal cover, it is a product of $\mathbb{C}^{k}$ with compact Hermitian symmetric spaces. Without loss of generality we can assume that the first Chern class $c_{1}(M)$ is a positive multiple of the Kähler class. Then the KählerRicci flow will have singularity, say at $t=1$, and the normalized flow has long time existence. The re-scaling is given by $\hat{g}=\frac{1}{1-t} g$ and the re-parametrization is given by $s=-\log (1-t)$. Therefore, Theorem 2.2 has the following equivalent form.

Theorem 2.10. Let $M$ be a compact Kähler manifold as above. Let $g(x, t)$ be a solution to (2.1), and let $u(x, t)$ be a positive solution to (2.2). Assume that $\hat{g}(x, s)$ is the solution to the normalized Kähler Ricci flow. Then

$$
(\log u)_{\alpha \bar{\beta}}+\hat{R}_{\alpha \bar{\beta}}+\frac{1}{e^{s}-1} \hat{g}_{\alpha \bar{\beta}} \geq 0 .
$$

Here $\hat{R}_{\alpha \bar{\beta}}$ is the Ricci tensor of $\hat{g}$.

\section{Monotonicity formulae}

In this section we derive some monotonicity formulae as a direct corollary of the matrix Li-Yau-Hamilton estimate, as well as its trace, proved in the last section. In order to make the argument unified for both cases with and without Ricci flow, we work with the interpolation version (2.7). Let $(M, g(t))$ be the solution to the Ricci flow (2.5) and let $u(x, t)$ be the positive solution to (2.6).

The first result is the monotonicity of the partition function (also called Nash's entropy in [FIN]) defined by

$$
\tilde{\mathcal{N}}(g, u, t)=-\int_{M} u \log u d v-m \log (\pi t)-m .
$$

Simple computation shows that

$$
\frac{d \tilde{\mathcal{N}}}{d t}=\int_{M}\left(-\Delta \log u-\epsilon \mathcal{R}-\frac{m}{t}\right) u d \mu_{t} \leq 0 .
$$

Here $d \mu_{t}$ is the volume element of $g(t)$. The following result is a direct consequence of Corollary 2.8. 
Proposition 3.1. Let $(M, g(t))$ be a solution to (2.5) with nonnegative bisectional curvature. Let $H(x, y, t)$ be the fundamental solution to (2.6). Then

$$
\frac{d}{d t} \tilde{\mathcal{N}}(g, u, t) \leq 0
$$

for any positive solution $u$ to (2.6) and

$$
-\int_{M} H \log H d \mu_{t}-m \log (\pi t) \leq m
$$

with the equality holds for some positive $t$ if and only if the manifold is an expanding gradient soliton (isometric to $\mathbb{C}^{m}$ in the case $\epsilon=0$ ).

For the fixed Riemannian metric case the above was proved earlier in [N2] for the manifold with only nonnegative Ricci curvature. We believe that the result should hold for Ricci flow even without assumptions. But at this moment we can only prove it for Kähler-Ricci flow through the matrix LYH inequality, which assumes the nonnegativity of the bisectional curvature. The result above gives a characterization of expanding solitons using the partition function. The similar formulation for the shrinking solitons also works for the partition functions related to Perelman's entropy formula $[\mathbf{P}]$. Namely, consider the backward Ricci flow $\frac{\partial}{\partial \tau} g_{i j}=2 R_{i j}$ on $M \times\left[0, \tau_{0}\right]$, where $M$ is a Riemannian manifold of real dimension $n$. Let $u(x, \tau)$ be a solution to the backward adjoint heat equation

$$
\left(\frac{\partial}{\partial \tau}-\Delta+\mathcal{R}\right) u(x, \tau)=0 .
$$

Similarly one can define

$$
\tilde{\mathcal{N}}(g, u, \tau)=-\int_{M} u \log u d \mu_{\tau}-\frac{n}{2} \log (4 \pi \tau)-\frac{n}{2} .
$$

In Proposition 1.2 of $[\mathbf{P}]$, Perelman proved that

$$
\frac{d \tilde{\mathcal{N}}}{d \tau}=\int_{M}\left(-\Delta \log u+\mathcal{R}-\frac{n}{2 \tau}\right) u d \mu_{\tau} \leq 0
$$

The dual version of Proposition 3.1 is stated as follows.

Proposition 3.2. Let $H(x, y, \tau)$ be the fundamental solution to the adjoint heat equation. Then

$$
-\int_{M} H \log H d \mu_{\tau}-\frac{n}{2} \log (4 \pi \tau) \leq \frac{n}{2}
$$

with equality holds (or in (3.5)) for some positive $\tau$ if and only if $\left(M, g_{i j}(\tau)\right)$ is a gradient shrinking soliton. 
Proof. Since we do not have Corollary 2.8 in this case we need other arguments. In fact, tracing the equality case of the proof of Proposition 1.2 of $[\mathbf{P}]$ we have that $R_{i j}-\nabla_{i} \nabla_{j} \log H$ is diagonal. Namely $R_{i j}-$ $\nabla_{i} \nabla_{j} \log H=\frac{\mathcal{R}-\Delta \log H}{n} g_{i j}$. On the other hand, the equality in (3.5) further implies that $R_{i j}-\nabla_{i} \nabla_{j} \log H=\frac{1}{2 \tau} g_{i j}$.

q.e.d.

In [N2], the relation between the value of $\tilde{\mathcal{N}}(t)$ (as well as the linear entropy functional, also denoted by $\mathcal{W}$ ) as $t \rightarrow \infty$ and the asymptotic volume ratio at infinity (also called the 'cone angle') was proved for the linear heat equation. The similar relation, between the $\kappa$-constant in the $\kappa$-non-collapsing of volume defined in $[\mathbf{P}]$ and the large time limit of the partition function $\tilde{\mathcal{N}}(g, u, \tau)$ (as well as the entropy functional $\mathcal{W}$ ) should also be true for ancient solutions to Ricci flow.

Another application of Theorem 2.2 is an entropy monotonicity for the ancient solutions. Let $(M, g(t))$ be an ancient solution to $(2.5)$ and let $u$ be a positive solution to (2.6), both defined on $M \times(-\infty, 0]$. Theorem 2.2 implies that

$$
(\log u)_{\alpha \bar{\beta}}+R_{\alpha \bar{\beta}} \geq 0 .
$$

From this one can easily see that

$$
\mathcal{N}(g, u, t):=-\int_{M} u \log u d \mu_{t}
$$

is monotone non-increasing.

If $M$ is compact, one can obtain such $u$ by taking limit of solutions with initial data at $t=-k$ as in [FIN] (where the immortal solution is studied). More precisely, let $u_{k}(x, t)$ be a solution to (2.6) with $u(x,-k)=\frac{1}{V(-k)}$. Letting $k \rightarrow \infty$, one can extract a limit $u_{\infty}>0$ (since $\int_{M} u_{k} \equiv 1$ and $\left.u_{k}>0\right)$ which is defined on $(-\infty, 0]$. In this case $\int_{M} u_{\infty} d v=1$. Applying the Jensen's inequality one has that

$$
\mathcal{N}\left(g, u_{\infty}, t\right) \leq \log V(t) .
$$

The right hand side is another monotone non-increasing quantity along the flow. When $M$ is complete noncompact one can obtain such a $u$ similarly by solving $u_{k}(x, t)$ with initial condition at $t=-k$ and anchoring $u_{k}(o,-1)=1$, where $o \in M$ is a fixed point. In fact one can even get $u_{k}$ integrable by taking it to be scalar multiple of the fundamental solution (with initial data being the delta function at $t=$ $-k)$.

As the other application considered in this section, we shall derive the heat kernel comparison theorem and Huisken type $([\mathbf{H u}],[\mathbf{E 2}])$ monotonicity formula for the analytic subvarieties in $M$. We start with the case $\epsilon=0$ since the results seem to be more useful at this moment.

Theorem 3.3. Let $M$ be a complete Kähler manifold with nonnegative bisectional curvature. Let $H(x, y, t)$ be the fundamental solution of 
the heat equation. Let $\mathcal{V} \subset M$ be any complex subvariety of dimension $s$. Let $K_{\mathcal{V}}(x, y, t)$ be the fundamental solution of heat equation on $\mathcal{V}$. Then

$$
K_{\mathcal{V}}(x, y, t) \leq(\pi t)^{m-s} H(x, y, t), \text { for any } x, y \in \mathcal{V} .
$$

If the equality holds, then $\mathcal{V}$ is totally geodesic. Furthermore, if $\tilde{M}$ is the universal cover of $M$ with covering map $\pi$ and $\tilde{\mathcal{V}}=\pi^{-1}(\mathcal{V})$, then $\tilde{M}=\tilde{M}_{1} \times \mathbb{C}^{k}$ for some Kähler manifold $\tilde{M}_{1}$ which does not contain any Euclidean factors, with $k \geq m-s$. Moreover $\tilde{\mathcal{V}}=\tilde{M}_{1} \times \mathbb{C}^{l}$ with $l<k$.

(ii)

$$
\frac{d}{d t} \int_{\mathcal{V}}(\pi t)^{m-s} H(x, y, t) d A_{\mathcal{V}}(y) \geq 0, \text { for any } x \in M .
$$

Similarly, if the equality holds for some $x \in M$ at some positive time $t$, then $\tilde{M}=\tilde{M}_{1} \times \mathbb{C}^{k}$ with $k \geq m-s$.

Proof. For any smooth point $y \in \mathcal{V}$, choose a complex coordinate $\left(z_{1} \cdots, z_{m}\right)$ such that $\left(z_{1}, \cdots, z_{s}\right)$ for a coordinate for $\mathcal{V}$ near $y$. Let $i, j, k, \cdots$ be the index of the coordinate functions of $\mathcal{V}$ and $a, b, c, \cdots$ be the index of coordinate function in the normal directions. Then we compute

$$
\begin{aligned}
& \left(\Delta_{\mathcal{V}}^{(y)}-\frac{\partial}{\partial t}\right)\left((\pi t)^{m-s} H(x, y, t)\right) \\
& =\left(\Delta_{M}-g^{a \bar{b}} \nabla_{a} \nabla_{\bar{b}}-\frac{\partial}{\partial t}\right)\left(t^{m-s} H(x, y, t)\right) \\
& =(\pi t)^{m-s}\left(\Delta_{M} H-H_{t}-g^{a \bar{b}} \nabla_{a} \nabla_{\bar{b}} H-\frac{m-s}{t} H\right) \\
& =(\pi t)^{m-s}\left(-\nabla_{a} \nabla_{\bar{b}} H+\frac{\nabla_{a} H \nabla_{\bar{b}} H}{H}-\frac{1}{t} H g_{a \bar{b}}\right) g^{a \bar{b}} \\
& \quad-(\pi t)^{m-s} \frac{\left|\nabla^{\perp} H\right|^{2}}{H} .
\end{aligned}
$$

By Theorem 2.2 we have that

$$
\left(\Delta_{\mathcal{V}}^{(y)}-\frac{\partial}{\partial t}\right)\left((\pi t)^{m-s} H(x, y, t)\right) \leq 0 .
$$

Notice that for $x \in \mathcal{V},\left.\lim _{t \rightarrow 0}(\pi t)^{m-s} H(x, y, t)\right|_{\mathcal{V}}=\delta_{x}(y)$. This proves that (3.6) by the maximum principle, (3.7) by the integrating (3.8) on $\mathcal{V}$. (For the case $\mathcal{V}$ is singular, one can refer to $[\mathbf{L T}]$ for the justification on the validity of the integration by parts.)

If the equality holds in (3.6) then $(\pi t)^{m-s} H(x, y, t)$ satisfies the heat equation. Hence equality holds in (3.8) for any $x, y \in \mathcal{V}$. This implies 
that

$$
\left|\nabla^{\perp} \log H\right|^{2} \equiv 0, \text { for } x, y \in \mathcal{V}, t>0,
$$

which implies that $\left\langle\nabla r^{2}(x, y), \nu\right\rangle=0$ for any $x, y \in \mathcal{V}$, and any normal direction $\nu$. Here $r(x, y)$ is the distance function between $x$ and $y$ and we have used the fact that $\lim _{t \rightarrow 0}-t \log H(x, y, t)=r^{2}(x, y)$. See [CLY1], for example. This implies that for any smooth point $x \in \mathcal{V}$, any minimizing geodesic starting from $x$ lies totally inside $\mathcal{V}$. More precisely, let $\gamma_{v}(s)$ be a short minimizing geodesic emitting from $x$ with $\gamma_{v}^{\prime}(0)=v$ such that $v \in T_{x} \mathcal{V}$. Denote by $h$ be the distance function from $\mathcal{V}$. Then

$$
\begin{aligned}
\frac{d}{d s} h\left(\gamma_{v}(s)\right) & =2 \operatorname{Re}\left\langle\nabla h, \gamma_{v}^{\prime}(s)\right\rangle \\
& =\frac{1}{s} 2 \operatorname{Re}\left\langle\nabla h, s \gamma_{v}^{\prime}(s)\right\rangle \\
& =\frac{1}{s} \operatorname{Re}\left\langle\nabla h, \nabla r^{2}\left(x, \gamma_{v}(s)\right)\right\rangle=0 .
\end{aligned}
$$

Here $\nabla h=\nabla^{\alpha} h \frac{\partial}{\partial z^{\alpha}}$ and $\gamma_{v}^{\prime}(s)=\frac{d z^{\alpha}(s)}{d s} \frac{\partial}{\partial z^{\alpha}}$. Therefore, $\mathcal{V}$ is totally geodesic. This in particular shows that there are no singular points in $\mathcal{V}$, which rules out the possibility that $\mathcal{V}$ is a union of several totally geodesic submanifolds with singular intersections (in which case the heat kernel comparison (3.6) has strictly inequality). Moreover, by lifting the computation to the universal cover we can assume that $M$ is simply-connected. Then the manifold $M$ splits by Theorem 0.1 of [NT2], more precisely, Theorem 2.1 and Corollary 2.1 of [NT2], since $N_{\alpha \bar{\beta}}=\nabla \alpha \nabla_{\bar{\beta}} H-\frac{\nabla_{\alpha} H \nabla_{\bar{\beta}} H}{H}+\frac{1}{t} H g_{\alpha \bar{\beta}} \geq 0$ and its null space is at least of $m-s$ dimension, by (3.8), for any $x, y \in \mathcal{V}$ and $t>0$. Notice that $N_{\alpha \bar{\beta}}$ does not satisfies the linear Lichnerowicz heat equation. However, the inequality (2.21), satisfied by $N_{\alpha \bar{\beta}}$ (since $\left.\epsilon=0\right)$, is enough for the argument in the proof of Corollary 2.1 of [NT2]. Then $M=M_{1} \times M_{2}$ such that the tangent space of $M_{2}$ consists of the null space of $N_{\alpha \bar{\beta}}$. That the factor $M_{2}$ is isometric to $\mathbb{C}^{m-s}$ follows from the same argument as in the proof of Corollary 2.8, since $-\nabla_{a} \nabla_{\bar{b}} H+\frac{\nabla_{a} H \nabla_{\bar{b}} H}{H}-\frac{1}{t} H g_{a \bar{b}} \equiv 0$. (One can also use Corollary 1.3 of $[\mathbf{N 2}]$ on p. 331.) More precisely, if we write a point $x \in M$ as $x=\left(x_{1}, x_{2}\right)$ according to the splitting, we can write the heat kernel $H(x, y, t)=H_{1}\left(x_{1}, y_{1}, t\right) H_{2}\left(x_{2}, y_{2}, t\right)$. Then on $M_{2}$ we have that $\left(\log H_{2}\left(x_{2}, y_{2}\right)\right)_{\alpha \bar{\beta}}+\frac{1}{t} g_{\alpha \bar{\beta}}=0$ by the definition of the splitting. Therefore one can apply Corollary 2.8 to conclude that $M_{2}=\mathbb{C}^{k}$. If (3.7) holds equality for some $x \in M$, it implies that the right hand side of (3.8) is zero. Then the argument above also applies. Note that we may not have $\mathcal{V}$ totally geodesic since $x$ may be a singular point.

q.e.d. 


\section{Remark 3.4.}

(1) In the case $\mathcal{V}$ is not smooth, the existence of $K(x, y, t)$ was justified in the work of Li and Tian $[\mathbf{L T}]$. They also obtained a similar upper bound estimate as (3.6) for the special case $M=\mathbb{P}^{m}$ (equipped with the Fubini-Study metric) using a very different method. Their method produces better upper bound for the special case $M=\mathbb{P}^{m}$. Our estimate here works for general Kähler manifolds with nonnegative bisectional curvature.

(2) The similar heat kernel comparison was first proved in [CLY2] for minimal submanifolds in space forms by a quite different method. (The method of $[\mathbf{L T}]$ is closely related to that of [CLY2].) The result in Theorem 3.3 is more general than [CLY2] in the sense that it holds for any Kähler manifolds with nonnegative sectional curvature instead of space forms. However, it is also more restrictive since it only applies to analytic subvarieties (which are known to be area-minimizing).

(3) In part (ii) of Theorem 3.3, the manifold $\tilde{M}_{1}$ may not be $\tilde{\mathcal{V}}$. This could happen, for example, in the case $M=\mathbb{C}^{m}$ and $\mathcal{V}$ is a union of two hyper-planes and $x$ lies on the intersection subvariety. If we further assume that equality holds for all smooth points $x \in \mathcal{V}$, we do have the same conclusion as part (i).

(4) The monotonicity (3.7) is enough for applications in [N4]. Namely, one can prove the comparison results on the dimensions of polynomial growth holomorphic function spaces obtained in [N4], using (3.7) instead of the other LYH inequality proved therein. In fact, one can derive the results in $[\mathbf{N} 4]$ through the following corollary, which is a special case of Theorem 3.3 (or Corollary 3.8) of [N4]. It is, however, enough for the applications considered in [N4].

Corollary 3.5. Let $f$ be a holomorphic function. Let $\mathcal{V}=Z(f)$, the zero locus of $f$. Denote

$$
w(x, t)=\int_{M} H(x, y, t) \Delta \log |f|^{2}(y) d \mu(y) .
$$

Then

$$
t w(x, t)=(\pi t) \int_{\mathcal{V}} H(x, y, t) d A_{\mathcal{V}}(y)
$$

Moreover

$$
\frac{\partial}{\partial t}(t w(x, t)) \geq 0
$$

If the equality holds for some point $x \in M$ and some positive time $t$, then the universal cover (of $M$ ) $\tilde{M}$ splits at least a factor of $\mathbb{C}$.

Proof. Notice that $\Delta=g^{\alpha \bar{\beta}} \frac{\partial}{\partial z^{\alpha} \partial z^{\beta}}$, which differs by a factor of 4 from [N4]. Let $f$ be a holomorphic function defined on $M$. Here we do 
require some growth condition on $f$. For example, it is sufficient if $f$ is of polynomial growth or is of finite order in the sense of Hadamard (see (3.1) of [N4] for precise definition). We can write

$$
v(x, t)=\int_{M} H(x, y, t) \log |f|^{2}(y) d \mu(y)
$$

as a solution to the heat equation with initial value $\log |f|^{2}(y)$. The requirement on $f$ is to make such a representation formula of $v(x, t)$ meaningful. If $w(x, t)$ (we abuse the notation here) is defined to be $\frac{\partial}{\partial t} v(x, t)$ as in $[\mathbf{N} \mathbf{4}]$, then it is a solution to the heat equation with initial data $\Delta \log |f|^{2}$. By the definition of $w(x, t)$ in (3.10) it is easy to see that $w(x, t)$ is also a solution to the heat equation with initial data given by the measure $\Delta \log |f|^{2}$. Hence $w(x, t)=\frac{\partial}{\partial t} v(x, t)$. By the Poincaré-Lelong formula we know that

$$
\begin{aligned}
& (\pi t) \int_{M} H(x, y, t)\left(\frac{\sqrt{-1}}{2 \pi} \partial \bar{\partial} \log |f|^{2}(y)\right) \wedge \frac{\omega^{m-1}}{(m-1) !} \\
& =(\pi t) \int_{\mathcal{V}} H(x, y, t) d A_{\mathcal{V}}(y) .
\end{aligned}
$$

On the other hand, the direct calculation shows that the left hand side of the above equation is equal to

$$
\begin{aligned}
t \int_{M} H(x, y, t) \Delta \log |f|^{2}(y) \frac{\omega^{m}}{m !} & =t \int_{M} H(x, y, t) \Delta \log |f|^{2}(y) d \mu(y) \\
& =t w(x, t) .
\end{aligned}
$$

This proves the first statement of the corollary. The monotonicity (3.12) is just a special case (ii) of Theorem 3.3. The proof above also shows that $w(x, t)$ has the same meaning as in Lemma 3.1 and Theorem 3.1, Theorem 4.1 of $[\mathbf{N 4}]$. $\quad$ q.e.d.

The case with Ricci flow, namely $\epsilon=1$, can be formulated similarly. In order to make it precise we have to explain some notations. For timedependent metrics deformed by the Kähler-Ricci flow equation (2.1), we call $H\left(x, t ; y, t_{0}\right)$ (with $t \geq t_{0}$ ) a fundamental solution to the forward conjugate heat equation. If it satisfies (2.2) (with respect to $x$ ) and $\lim _{t \rightarrow t_{0}} H\left(x, t ; y, t_{0}\right)=\delta_{y}(x)$, it is easy to see that (2.2) is conjugate to the backward heat equation $\left(\frac{\partial}{\partial t}+\Delta\right) v(x, t)=0$. Therefore if we denote by $H^{*}\left(x, t ; y, t_{0}\right)$ (with $\left.t \leq t_{0}\right)$ the fundamental solution to $\frac{\partial}{\partial t}+\Delta$, then a well-known duality asserts that $H\left(x, t ; y, t_{0}\right)=H^{*}\left(y, t_{0} ; x, t\right)$. From this it is easy to see that for any solution $u(x, t)$ to the forward conjugate heat equation we have the representation formula:

$$
u(x, t)=\int_{M} u(y, 0) H^{*}(y, 0 ; x, t) d \mu_{0}(y)=\int_{M} H(x, t ; y, 0) u(y, 0) d \mu_{0}(y) .
$$


For most of our discussion we assume that $t_{0}=0$ as above and we also just write $H(x, t ; y, 0)$ as $H(x, y, t)$. Adapting the notation from the proof of Theorem 3.3, the restricted Ricci flow equation on a subvariety $\mathcal{V}$ is $\frac{\partial}{\partial t} g_{i \bar{j}}=-2 R_{i \bar{j}}$. Let $\mathcal{R}_{\mathcal{V}}=g^{i \bar{j}} R_{i \bar{j}}$. Then the restricted forward conjugate heat equation is

$$
\left(\frac{\partial}{\partial t}-\Delta_{\mathcal{V}}\right) v(x, t)=\mathcal{R}_{\mathcal{V}}(x, t) v(x, t) .
$$

We denote by $K_{\mathcal{V}}(x, y, t)$ (here again we assume $t_{0}=0$ and use $K_{\mathcal{V}}(x, y, t)$ instead of $\left.K_{\mathcal{V}}(x, t ; y, 0)\right)$ the fundamental solution of the restricted forward conjugate heat equation. We have the following comparison and monotonicity result.

Theorem 3.6. Let $M$ be a complete Kähler manifold with bounded nonnegative bisectional curvature. Let $H(x, y, t)$ be a fundamental solution to the forward conjugate heat equation on $M$. Let $\mathcal{V}$ be a complex subvariety of $M$ of dimension s. Let $K \mathcal{V}(x, y, t)$ be the fundamental solution to the restricted forward conjugate heat equation (with respect to the induced metrics) on $\mathcal{V}$. Then we have (3.6) and (3.7). Moreover, the equality (for positive $t$ ), in either case, implies that the universal cover $($ of $M) \tilde{M}$ has the splitting $\tilde{M}=\tilde{M}_{1} \times \mathbb{E}^{k}$, where $\mathbb{E}^{k}$ is a gradient expanding Kähler-Ricci soliton of dimension $k \geq m-s$.

Remark 3.7. One can think of (3.7) as a dual version of Perelman's monotonicity of the reduced volume since the reduced volume in the Section 7 of $[\mathbf{P}]$ is, in a sense, a 'weighted volume' of $M$ (with weight being the fundamental solution (to the backward conjugate heat equation) of a 'potentially infinite dimensional manifold' restricted to $M$, as explained in Section 6 of $[\mathbf{P}])$, while here the monotonicity is on the 'weighted volume' of complex submanifolds with weight being the fundamental solution (of the forward conjugate heat equation) of $M$ restricted to the submanifold. The reduced volume monotonicity of Perelman has important applications in the study of Ricci flow. We expect that (3.7) will have some applications in understanding the relation between Kähler-Ricci flow and the complex geometry of analytic subvarieties.

Taking the trace of the matrix estimate in Theorem 2.2 and integrating along the space-time path as in $[\mathbf{L Y}]$, we can have the following Harnack estimates for the positive solutions to the forward conjugate heat equation. This gives a sharp version of the previous mentioned rough estimate of $[\mathbf{G u}]$.

Corollary 3.8. Let $(M, g(t))$ be a solution to Ricci flow (2.1) and $u(x, t)$ be a positive solution to (2.2). Then

$$
\frac{|\nabla u|^{2}}{u^{2}}-\frac{u_{t}}{u}+\frac{m}{t} \geq 0
$$


and for any $t_{2}>t_{1}$,

$$
u\left(x_{2}, t_{2}\right) t_{2}^{m} \geq u\left(x_{1}, t_{1}\right) t_{1}^{m} \exp \left(-\inf _{\gamma} \int_{t_{1}}^{t_{2}}\left|\gamma^{\prime}(t)\right|^{2} d t\right) .
$$

Here $\gamma(t)$ is a path with $\gamma\left(t_{1}\right)=x_{1}$ and $\gamma\left(t_{2}\right)=x_{2}$.

Note that we do not have the factor 4 due to our choice of $\Delta$ and that the gradient $|\nabla f|^{2}$ is defined to be $g^{\alpha \bar{\beta}} f_{\alpha} f_{\bar{\beta}}$. Here $\left|\gamma^{\prime}(t)\right|^{2}=$ $g_{\alpha \bar{\beta}} \frac{d z^{\alpha}}{d t} \frac{d z^{\bar{\beta}}}{d t}$. We list this consequence here since it implies the monotonicity of $t^{m} u(x, t)$.

Finally we should point out that, as in $[\mathbf{H 3}]$, the matrix LYH inequality also implies the monotonicity of the weighted energy of a holomorphic mappings from $M$ (into any Kähler manifolds), as well as the monotonicity of the weighted energy for Hermitian-Einstein flow on any holomorphic vector bundle over $M$. For example, if $F$ is a holomorphic mapping from $M$ (into say another Kähler manifold $N$ ), then we have that

$$
\frac{d}{d t}\left((T-t) \int_{M}|\partial F|^{2} H\left(x, t ; x_{0}, T\right) d \mu\right) \leq 0
$$

where $|\partial F|^{2}=g^{\alpha \bar{\beta}} h_{i \bar{j}} F_{\alpha}^{i} F_{\bar{\beta}}^{\bar{j}}, H\left(x, t ; x_{0}, T\right)$ is the fundamental solution to the backward heat equation satisfying $\left(\frac{\partial}{\partial t}+\Delta\right) H\left(x, t ; x_{0}, T\right)=$ $\delta_{\left(x_{0}, T\right)}(x, t)$. Dually we also have that

$$
\frac{d}{d t}\left(t \int_{M}|\partial F|^{2} H\left(x, t ; x_{0}, 0\right) d \mu\right) \geq 0
$$

where $H\left(x, t ; x_{0}, 0\right)$ is the fundamental solution of the heat equation centered at $\left(x_{0}, 0\right)$.

\section{Interpolation between Perelman's entropy formula and the new LYH inequality}

The purpose of this section is two-folded. First we give a different proof of Theorem 2.2. The second purpose is to show that a by-product of this second proof also implies Perelman's monotonicity of entropy, as well as the energy. The computation in this section has its real version. See $[\mathbf{C L N}]$ for more details. The main computation is summarized in equation (4.7) below, which is called a pre- $L Y H$ equality. The equation (4.7) can also be viewed as a matrix version of Perelman's entropy monotonicity formula. In a sense, one can think that the matrix LYH inequality proved in Section 2 is dual to Perelman's entropy formula in Section 3 of $[\mathbf{P}]$. 
Consider the Kähler-Ricci flow:

$$
\frac{\partial}{\partial \tau} g_{\alpha \bar{\beta}}=\epsilon R_{\alpha \bar{\beta}}
$$

where $\epsilon$ is a parameter and the conjugate heat equation:

$$
\left(\frac{\partial}{\partial \tau}-\Delta+\epsilon \mathcal{R}\right) u(x, \tau)=0 .
$$

When $\epsilon<0,(4.1)$ is a forward Ricci flow equation and (4.2) becomes a forward conjugate heat equation. The equations look different from those in Section 2 since in this section the case of $\epsilon<0$ corresponds to the forward Ricci flow and the case of $\epsilon>0$ corresponds to the backward Ricci flow. For example $\epsilon=1$ is exactly the setting for Perelman's entropy and energy monotonicity. Notice that (4.2) becomes the backward conjugate heat equation for $\epsilon=1$. For the positive solution $u(x, \tau)$ we define the $(1,1)$ tensor $Z_{\alpha \bar{\beta}}$ by

$$
Z_{\alpha \bar{\beta}}=-(\log u)_{\alpha \bar{\beta}}+\epsilon R_{\alpha \bar{\beta}} .
$$

Let $\Delta_{L}$ denote the Lichnerowicz Laplacian on $(1,1)$ tensors, which is defined by

$$
\Delta_{L} \eta_{\alpha \bar{\beta}}=\Delta \eta_{\alpha \bar{\beta}}+R_{\alpha \bar{\beta} \gamma \bar{\delta}} \eta_{\bar{\gamma} \delta}-\frac{1}{2}\left(R_{\alpha \bar{p}} \eta_{p \bar{\beta}}+\eta_{\alpha \bar{p}} R_{p \bar{\beta}}\right)
$$

for any Hermitian symmetric (1.1) tensor $\eta_{\alpha \bar{\beta}}$. It is known, see for example $[\mathbf{C 1}]$, that

$$
\left(\frac{\partial}{\partial \tau}-\Delta_{L}\right) R_{\alpha \bar{\beta}}=-(1+\epsilon) \Delta_{L} R_{\alpha \bar{\beta}}
$$

The direct calculation as in [NT1] Lemma 2.1, shows that

Lemma 4.1. For any $C^{2}$-function $f(x, \tau)$

$$
\left(\frac{\partial}{\partial \tau}-\Delta_{L}\right) f_{\alpha \bar{\beta}}=\left[\left(\frac{\partial}{\partial \tau}-\Delta\right) f\right]_{\alpha \bar{\beta}}
$$

Remark 4.2. A result similar to Lemma 4.1 holds for Ricci flow on Riemannian manifolds. (See $[\mathbf{C L N}]$ for details.)

Now with the help of Lemmas 4.1 and 2.3, using the equation

$$
\left(\frac{\partial}{\partial \tau}-\Delta_{L}\right)(\log u)=-\epsilon \mathcal{R}+|\nabla \log u|^{2}
$$


we can compute

$$
\begin{aligned}
& \left(\frac{\partial}{\partial \tau}-\Delta_{L}\right) Z_{\alpha \bar{\beta}} \\
& =\left(\epsilon \mathcal{R}-|\nabla \log u|^{2}\right)_{\alpha \bar{\beta}}-\epsilon(1+\epsilon)_{L} R_{\alpha \bar{\beta}} \\
& =\epsilon(\mathcal{R})_{\alpha \bar{\beta}}-\left(g^{\gamma \bar{\delta}}(\log u)_{\gamma}(\log u)_{\bar{\delta}}\right)_{\alpha \bar{\beta}}-\epsilon(1+\epsilon) \Delta_{L} R_{\alpha \bar{\beta}} \\
& =-R_{\alpha \bar{\beta} \gamma \bar{\delta}}(\log u)_{\bar{\gamma}}(\log u)_{\delta}-(\log u)_{\alpha \gamma}(\log u)_{\bar{\gamma} \bar{\beta}} \\
& \quad-(\log u)_{\alpha \bar{\gamma}}(\log u)_{\gamma \bar{\beta}}-\left[(\log u)_{\alpha \bar{\beta}}\right]_{\gamma}(\log u)_{\bar{\gamma}} \\
& \quad-\left[(\log u)_{\alpha \bar{\beta}}\right]_{\bar{\gamma}}(\log u)_{\gamma}-\epsilon^{2} \Delta_{L} R_{\alpha \bar{\beta}} .
\end{aligned}
$$

Hence

$$
\begin{aligned}
& \left(\frac{\partial}{\partial \tau}-\Delta_{L}\right) Z_{\alpha \bar{\beta}} \\
& =-\epsilon^{2}\left(\Delta R_{\alpha \bar{\beta}}+R_{\alpha \bar{\beta} \gamma \bar{\delta}} R_{\bar{\gamma} \delta}+\nabla_{\gamma} R_{\alpha \bar{\beta}}\left(\frac{1}{\epsilon} \nabla_{\bar{\gamma}} \log u\right)\right. \\
& \left.\quad+\nabla_{\bar{\gamma}} R_{\alpha \bar{\beta}}\left(\frac{1}{\epsilon} \nabla_{\gamma} \log u\right)+R_{\alpha \bar{\beta} \gamma \bar{\delta}}\left(\frac{1}{\epsilon} \nabla_{\bar{\gamma}} \log u\right)\left(\frac{1}{\epsilon} \nabla_{\delta} \log u\right)\right) \\
& \quad+\epsilon^{2} R_{\alpha \bar{\gamma}} R_{\gamma \bar{\beta}}-(\log u)_{\alpha \gamma}(\log u)_{\bar{\gamma} \bar{\beta}}-(\log u)_{\alpha \bar{\gamma}}(\log u)_{\gamma \bar{\beta}} \\
& \quad+\nabla_{\gamma}\left(Z_{\alpha \bar{\beta}}\right) \nabla_{\bar{\gamma}} \log u+\nabla_{\bar{\gamma}}\left(Z_{\alpha \bar{\beta}}\right) \nabla_{\gamma} \log u .
\end{aligned}
$$

Regrouping terms yields

$$
\begin{aligned}
& \left(\frac{\partial}{\partial \tau}-\Delta_{L}\right) Z_{\alpha \bar{\beta}} \\
& =-\epsilon^{2}\left(\Delta R_{\alpha \bar{\beta}}+R_{\alpha \bar{\beta} \gamma \bar{\delta}} R_{\bar{\gamma} \delta}+\nabla_{\gamma} R_{\alpha \bar{\beta}}\left(\frac{1}{\epsilon} \nabla_{\bar{\gamma}} \log u\right)\right. \\
& \left.\quad+\nabla_{\bar{\gamma}} R_{\alpha \bar{\beta}}\left(\frac{1}{\epsilon} \nabla_{\gamma} \log u\right)+R_{\alpha \bar{\beta} \gamma \bar{\delta}}\left(\frac{1}{\epsilon} \nabla_{\bar{\gamma}} \log u\right)\left(\frac{1}{\epsilon} \nabla_{\delta} \log u\right)\right) \\
& \quad-(\log u)_{\alpha \gamma}(\log u)_{\bar{\gamma} \bar{\beta}}+\nabla_{\gamma}\left(Z_{\alpha \bar{\beta}}\right) \nabla_{\bar{\gamma}} \log u+\nabla_{\bar{\gamma}}\left(Z_{\alpha \bar{\beta}}\right) \nabla_{\gamma} \log u \\
& \quad+\frac{1}{2} Z_{\alpha \bar{\gamma}}\left(\epsilon R_{\gamma \bar{\beta}}+(\log u)_{\gamma \bar{\beta}}\right)+\frac{1}{2}\left(\epsilon R_{\alpha \bar{\gamma}}+(\log u)_{\alpha \bar{\gamma}}\right) Z_{\gamma \bar{\beta}} .
\end{aligned}
$$

Let

$$
\tilde{Z}_{\alpha \bar{\beta}}=Z_{\alpha \bar{\beta}}-\frac{1}{\tau} g_{\alpha \bar{\beta}}
$$

and

$$
\begin{aligned}
Y_{\alpha \bar{\beta}}= & \Delta R_{\alpha \bar{\beta}}+R_{\alpha \bar{\beta} \gamma \bar{\delta}} R_{\bar{\gamma} \delta}+\nabla_{\gamma} R_{\alpha \bar{\beta}}\left(\frac{1}{\epsilon} \nabla_{\bar{\gamma}} \log u\right) \\
& +\nabla_{\bar{\gamma}} R_{\alpha \bar{\beta}}\left(\frac{1}{\epsilon} \nabla_{\gamma} \log u\right)+R_{\alpha \bar{\beta} \gamma \bar{\delta}}\left(\frac{1}{\epsilon} \nabla_{\bar{\gamma}} \log u\right)\left(\frac{1}{\epsilon} \nabla_{\delta} \log u\right) .
\end{aligned}
$$


Notice that $\tilde{Y}_{\alpha \bar{\beta}}$, defined after $(2.20)$ in Section 2, is related to $Y_{\alpha \bar{\beta}}$ above through the equation $\tilde{Y}_{\alpha \bar{\beta}}=Y_{\alpha \bar{\beta}}-\frac{R_{\alpha \bar{\beta}}}{\epsilon \tau}$ (remember that $-\epsilon$ here corresponding to $\epsilon$ in Section 2). From (4.6), we can derive the equation for $\tilde{Z}_{\alpha \bar{\beta}}$ as follows.

Lemma 4.3 (Chow-Ni).

$$
\begin{aligned}
\left(\frac{\partial}{\partial \tau}-\Delta_{L}\right) \tilde{Z}_{\alpha \bar{\beta}}= & \left(\frac{\partial}{\partial \tau}-\Delta_{L}\right) Z_{\alpha \bar{\beta}}+\frac{1}{\tau^{2}} g_{\alpha \bar{\beta}}-\frac{1}{\tau} \epsilon R_{\alpha \bar{\beta}} \\
= & -\left(\epsilon^{2} Y_{\alpha \bar{\beta}}-\frac{\epsilon}{\tau} R_{\alpha \bar{\beta}}\right)-(\log u)_{\alpha \gamma}(\log u)_{\bar{\gamma} \bar{\beta}} \\
& +\nabla_{\gamma}\left(\tilde{Z}_{\alpha \bar{\beta}}\right) \nabla_{\bar{\gamma}} \log u+\nabla_{\bar{\gamma}}\left(\tilde{Z}_{\alpha \bar{\beta}}\right) \nabla_{\gamma} \log u \\
& +\frac{1}{2} \tilde{Z}_{\alpha \bar{\gamma}}\left(\epsilon R_{\gamma \bar{\beta}}+(\log u)_{\gamma \bar{\beta}}\right) \\
& +\frac{1}{2}\left(\epsilon R_{\alpha \bar{\gamma}}+(\log u)_{\alpha \bar{\gamma}}\right) \tilde{Z}_{\gamma \bar{\beta}}-\frac{1}{\tau} \tilde{Z}_{\alpha \bar{\beta}} .
\end{aligned}
$$

Notice that $\tilde{Z}_{\alpha \bar{\beta}}=\frac{1}{u} \tilde{N}_{\alpha \bar{\beta}}$. With some labor one can check that (2.21) and (4.7) are equivalent. Namely one can derive one from the other, keeping in mind that $-\epsilon$ here corresponds $\epsilon$ in Section 2. One can also write (4.7) as

$$
\begin{aligned}
\left(\frac{\partial}{\partial \tau}-\Delta_{L}\right) \tilde{Z}_{\alpha \bar{\beta}}= & \left(\frac{\partial}{\partial \tau}-\Delta_{L}\right) Z_{\alpha \bar{\beta}}+\frac{1}{\tau^{2}} g_{\alpha \bar{\beta}}-\frac{1}{\tau} \epsilon R_{\alpha \bar{\beta}} \\
= & -\left(\epsilon^{2} Y_{\alpha \bar{\beta}}-\frac{\epsilon}{\tau} R_{\alpha \bar{\beta}}\right)-(\log u)_{\alpha \gamma}(\log u)_{\bar{\gamma} \bar{\beta}} \\
& +\nabla_{\gamma}\left(\tilde{Z}_{\alpha \bar{\beta}}\right) \nabla_{\bar{\gamma}} \log u+\nabla_{\bar{\gamma}}\left(\tilde{Z}_{\alpha \bar{\beta}}\right) \nabla_{\gamma} \log u \\
& +\frac{1}{2} \tilde{Z}_{\alpha \bar{\gamma}}\left(\epsilon R_{\gamma \bar{\beta}}+(\log u)_{\gamma \bar{\beta}}-\frac{1}{\tau} g_{\gamma \bar{\beta}}\right) \\
& +\frac{1}{2}\left(\epsilon R_{\alpha \bar{\gamma}}+(\log u)_{\alpha \bar{\gamma}}-\frac{1}{\tau} g_{\alpha \bar{\gamma}}\right) \tilde{Z}_{\gamma \bar{\beta}} .
\end{aligned}
$$

For $\epsilon<0$, applying Lemma 2.6, the result of [C1], we know that $\epsilon^{2} Y_{\alpha \bar{\beta}}-\frac{\epsilon}{\tau} R_{\alpha \bar{\beta}} \geq 0$ under the assumption that $M$ is a complete Kähler manifold with bounded nonnegative holomorphic bisectional curvature. Hence the tensor maximum principle and (4.8) imply that $\tilde{Z}_{\alpha \bar{\beta}} \leq 0$, which is equivalent to the statement of Theorem 2.2. Namely, (4.7) does lead to another proof of Theorem 2.2.

Even though the computations (4.7) and (2.21) are essentially equivalent, (4.7) has the advantage that when $\epsilon=1$ it also implies Perelman's energy/entropy monotonicity formulae. The following is a more detailed computation of this claim. Let $f=-\log u, Z=g^{\alpha \bar{\beta}} Z_{\alpha \bar{\beta}}$. Tracing (4.6) 
gives

$$
\begin{aligned}
\left(\frac{\partial}{\partial \tau}-\Delta\right) Z= & -\epsilon R_{\bar{\alpha} \beta} Z_{\alpha \bar{\beta}}-\epsilon^{2} g^{\alpha \bar{\beta}} Y_{\alpha \bar{\beta}}-(f)_{\alpha \gamma}(f)_{\bar{\gamma} \bar{\alpha}}-\nabla_{\gamma} Z \nabla_{\bar{\gamma}} f \\
& -\nabla_{\bar{\gamma}} Z \nabla_{\gamma} f+Z_{\alpha \bar{\beta}}\left(\epsilon R_{\beta \bar{\alpha}}-f_{\beta \bar{\alpha}}\right)
\end{aligned}
$$

and

$g^{\alpha \bar{\beta}} Y_{\alpha \bar{\beta}}=\Delta \mathcal{R}+R_{\alpha \bar{\beta}} R_{\bar{\alpha} \beta}-\nabla_{\gamma} \mathcal{R}\left(\frac{1}{\epsilon} \nabla_{\bar{\gamma}} f\right)-\left(\frac{1}{\epsilon} \nabla_{\gamma} f\right) \nabla_{\bar{\gamma}} \mathcal{R}+R_{\alpha \bar{\beta}}\left(\frac{1}{\epsilon} f_{\bar{\alpha}}\right)\left(\frac{1}{\epsilon} f_{\beta}\right)$.

The following observation of Chow is also useful.

Lemma 4.4 (Chow). In the case $\epsilon=1$, we have that

$$
\int_{M}\left(g^{\alpha \bar{\beta}} Y_{\alpha \bar{\beta}}\right) u d \mu=\int_{M}\left(R_{\alpha \bar{\beta}}\left(R_{\bar{\alpha} \beta}+f_{\bar{\alpha} \beta}\right)\right) u d \mu .
$$

Proof. The claim follows from the integration by parts and the second Bianchi identity $\mathcal{R}_{\gamma}=R_{\gamma \bar{\alpha}, \alpha}$.

q.e.d.

Remark 4.5. Please refer to $[\mathbf{C L N}]$ for the Riemannian version of the above identity.

Recall the definition of energy $\mathcal{F}$.

$$
\mathcal{F}(g, u, \tau)=\int_{M}\left(\frac{|\nabla u|^{2}}{u}+\mathcal{R} u\right) d \mu .
$$

Then (4.9) (with $\epsilon=1$ ) and Lemma 4.4 imply the the following result.

Proposition 4.6 (Perelman).

$$
\frac{d}{d \tau} \mathcal{F}(g, u, \tau)=-\int_{M}\left(\left|R_{\alpha \bar{\beta}}+f_{\alpha \bar{\beta}}\right|^{2}+\left|f_{\alpha \beta}\right|^{2}\right) u d \mu .
$$

Note that this is nothing but the energy monotonicity formula of Perelman in Section 1 of $[\mathbf{P}]$. Below we show in more detail that it follows from (4.9).

Proof of Proposition 4.6. Let $\epsilon=1$ in (4.9) and we have that

$\left(\frac{\partial}{\partial \tau}-\Delta\right) Z=-g^{\alpha \bar{\beta}} Y_{\alpha \bar{\beta}}-(f)_{\alpha \gamma}(f)_{\bar{\gamma} \bar{\alpha}}-\nabla_{\gamma} Z \nabla_{\bar{\gamma}} f-\nabla_{\bar{\gamma}} Z \nabla_{\gamma} f-Z_{\alpha \bar{\beta}} f_{\beta \bar{\alpha}}$.

Now the result follows from direct computation of

$$
\frac{d}{d \tau} \mathcal{F}(g, u, \tau)=\frac{d}{d \tau} \int_{M} Z u d \mu
$$

by applying Lemma 4.4 .

q.e.d. 
Similarly, if we trace (4.7) and denote $\tilde{Z}=g^{\alpha \bar{\beta}} \tilde{Z}_{\alpha \bar{\beta}}$, we have that

$$
\begin{aligned}
\left(\frac{\partial}{\partial \tau}-\Delta\right) \tilde{Z}= & -\epsilon R_{\bar{\alpha} \beta} \tilde{Z}_{\alpha \bar{\beta}}-\epsilon^{2} g^{\alpha \bar{\beta}} Y_{\alpha \bar{\beta}}+\frac{\epsilon}{\tau} \mathcal{R}-(f)_{\alpha \gamma}(f)_{\bar{\gamma} \bar{\alpha}} \\
& -\nabla_{\gamma} \tilde{Z} \nabla_{\bar{\gamma}} f-\nabla_{\bar{\gamma}} \tilde{Z} \nabla_{\gamma} f \\
& +\tilde{Z}_{\alpha \bar{\beta}}\left(\epsilon R_{\beta \bar{\alpha}}-f_{\beta \bar{\alpha}}\right)-\frac{1}{\tau} \tilde{Z} .
\end{aligned}
$$

For $\epsilon=1$, integration by parts as before gives

$$
\frac{d}{d \tau} \int_{M} \tilde{Z} u d \mu_{\tau}=-\int_{M}\left(\left|\tilde{Z}_{\alpha \bar{\beta}}\right|^{2}+\left|f_{\alpha \beta}\right|^{2}\right) u d \mu_{\tau}-\frac{2}{\tau} \int_{M} \tilde{Z} u d \mu_{\tau}
$$

The above equation is equivalent to Perelman's entropy monotonicity formula due to the following consideration. Let

$$
\tilde{\mathcal{N}}(g, u, \tau):=-\int_{M} u \log u d \mu_{\tau}-m \log (\pi \tau)-m .
$$

Then Perelman's entropy

$$
\mathcal{W}(g, u, \tau)=\int_{M}\left[\tau\left(2 \Delta \bar{f}-|\nabla \bar{f}|^{2}+\mathcal{R}+\bar{f}-2 m\right] u d \mu_{\tau},\right.
$$

where $\bar{f}=-\log u-m \log (\pi \tau)$, can be expressed as

$$
\mathcal{W}(g, u, \tau)=\frac{d}{d \tau}(\tau \tilde{\mathcal{N}})=\tau \int_{M} \tilde{Z} u d \mu_{\tau}+\tilde{\mathcal{N}}
$$

Therefore

$$
\begin{aligned}
\frac{d}{d \tau} \mathcal{W} & =\tau \frac{d}{d \tau} \int_{M} \tilde{Z} u d \mu_{\tau}+2 \int_{M} \tilde{Z} u d \mu_{\tau} \\
& =-\tau \int_{M}\left(\left|\tilde{Z}_{\alpha \bar{\beta}}\right|^{2}+\left|f_{\alpha \beta}\right|^{2}\right) u d \mu_{\tau}
\end{aligned}
$$

which is nothing but the entropy formula of Perelman in $[\mathbf{P}]$ Section 3.

As pointed out in $[\mathbf{P}]$, there exists a statistical mechanics analogy of Perelman's entropy. If we identify the quantities above with the notation of [Ev2], Chapter I and VII, $\tau$ is the temperature; $-\tilde{\mathcal{N}}$ defined above is the $\log$ of the distribution function in $[\mathbf{E v 2}] ;-\mathcal{W}$ is the entropy $S$ in [Ev2]; $\tau \tilde{\mathcal{N}}$ is the free energy $F ; \frac{\partial \tilde{\mathcal{N}}}{\partial\left(\frac{1}{\tau}\right)}$ is the energy $E$ in $[\mathbf{E v 2}]$ (which is nonnegative by Proposition 1.2 of $[\mathbf{P}]$ ) and the first equation of (4.15) is just the well-known equation $S=-\frac{\partial F}{\partial \tau}$ from thermodynamics. The negation of the right hand side of (4.16), measuring the deviation from a shrinking soliton, is the heat capacity of [Ev2], after multiplying it by $2 \tau$. The entropy formula (4.16) implies the concavity of $S$ in $E$, one of the defining properties for the entropy. This analogy also holds for the solution to the heat equation with respect to a fixed Riemannian metric with nonnegative Ricci curvature [N3]. 


\section{Remark 4.7.}

(1) For the case of $\epsilon=0$, a computation similar to the above gives the monotonicity formula in [N3]. The strange thing is that one can not get a nice monotonicity formula in the case $\epsilon \neq-1$ or 0 . Namely, the interpolation formula (4.6)/(4.7) does not give nice interpolation for energy/entropy after integration on $M$. One can view $(4.6) /(4.7)$ as a matrix version of the energy/entropy monotonicity formula for $\epsilon>0$. This partially answers one of the questions raised in the end of [N3] (still not satisfactory though). On the other hand, when $\epsilon<0$, one can not get entropy monotonicity out of (4.7). Instead we have a pointwise LYH inequality. However, for $\epsilon=0$, both entropy and the differential Harnack follows from (4.6) (see $[\mathbf{N 3}]$ ). The above discussion indicates that our new matrix LYH inequality is dual to the entropy monotonicity of Perelman in some sense and there may perhaps be certain profound duality behind the scene.

(2) Another puzzling point is that so far we have not been able to verify a matrix LYH inequality analogue to Theorem 2.2 for the Ricci flow on Riemannian manifolds, even though the above computation (4.7) holds for $\epsilon>0$ for the Ricci flow on Riemannian manifolds (which was carried out first in $[\mathbf{C L N}]$ ). In short, the interpolation between positive and negative $\epsilon$ by now only works in Kähler category. The validity of the Riemannian case is pending on the verification of a new matrix LYH estimate similar to Hamilton's famous work [H1]. Please see Remark 6.4 for further details.

\section{A local monotonicity formula and its applications}

This section is inspired by the works of Ecker [E1], [E2]. Let $M$ be a complete Kähler manifold with nonnegative bisectional curvature (unless specified otherwise). In this section we study the localization of the previous established monotonicity (in Section 3) for a fixed Kähler metric. (We leave the Kähler-Ricci flow case to a later discussion.) In [E1], the localized monotonicity formula is proved for mean curvature flow in Euclidean spaces. Since we are dealing with curved spaces here, we need some extra ingredients, which include Theorem 3.3 of Section 3, the complex Hessian comparison theorem on distance functions proved recently in $[\mathbf{L W}]$ (see also $[\mathbf{C N}]$ ), and the well-known heat kernel estimates of Li-Yau on complete Riemannian manifolds with nonnegative Ricci curvature, which states that

$$
\frac{C^{-1}(n)}{V_{x}(\sqrt{t})} \exp \left(-\frac{r^{2}(x, y)}{3 t}\right) \leq H(x, y, t) \leq \frac{C(n)}{V_{x}(\sqrt{t})} \exp \left(-\frac{r^{2}(x, y)}{5 t}\right)
$$


for some $C(n)>0$, where $n$ is the real dimension of the manifold considered. As applications we prove an elliptic 'monotonicity principle' for complex subvarieties in $M$. It can then be applied to prove a manifold version of Stoll's theorem.

Before we prove a localized version of Theorem 3.3, we need to introduce some functions (notations). Let $\mathcal{V}$ be an analytic subvariety (of $M$ ) of complex dimension $s$. For simplicity of notation, let $H(x, y, \tau)$ be the fundamental solution of the heat equation $\left(\frac{\partial}{\partial \tau}-\Delta\right) u(x, \tau)=0$. When $x, y \in \mathcal{V}$, we denote $(\pi \tau)^{m-s} H(x, y, \tau)$ by $H_{\mathcal{V}}(x, y, \tau)$. For any fixed $\left(x_{0}, t_{0}\right)$ with $x_{0} \in \mathcal{V}$, we denote $H_{\mathcal{V}}\left(x_{0}, y, t_{0}-t\right)$ by $\hat{H}_{\left(x_{0}, t_{0}\right), \mathcal{V}}(y, t)$. For any $\rho>0$, we also introduce a cut-off function

$$
\varphi_{\left(x_{0}, t_{0}\right), \rho}(y, t)=\left(1-\frac{r^{2}\left(x_{0}, y\right)+s\left(t-t_{0}\right)}{\rho^{2}}\right)_{+}
$$

where $f_{+}(x)=\max (f, 0)$ for any function $f$, and $r\left(x_{0}, y\right)$ is the distance function (of $M$ ) from $x_{0}$ to $y$. It is easy to see that $\varphi_{\left(x_{0}, t_{0}\right), \rho}$ is supported in $B_{x_{0}}\left(\sqrt{\rho^{2}-s\left(t-t_{0}\right)}\right)$.

The following simple lemma is useful.

Lemma 5.1. On $\mathcal{V}$

$$
\left(\frac{\partial}{\partial t}-\Delta_{\mathcal{V}}\right) \varphi_{\left(x_{0}, t_{0}\right), \rho}(y, t) \leq 0 .
$$

Here $\Delta_{\mathcal{V}}$ denotes the Laplacian operator with respect to the induced Kähler metric on $\mathcal{V}$ (strictly speaking the only regular part of $\mathcal{V}$ ).

Proof. For any $y \in \mathcal{V}$, choose a complex coordinate $\left(z_{1} \cdots, z_{m}\right)$ as in the proof of Theorem 3.3. Namely $z_{\alpha}=0$ on $\mathcal{V}$ for any $\alpha>s$. We also use the index convention as in the proof of Theorem 3.3. Namely, $1 \leq i, j, k, \cdots \leq s$ and $s+1 \leq a, b, c, \cdots \leq m$. Direct computation shows that

$$
\begin{aligned}
\left(\frac{\partial}{\partial t}-\Delta_{\mathcal{V}}\right)\left(s\left(t-t_{0}\right)+r_{x_{0}}^{2}(y)\right) & =s-g^{i \bar{j}}\left(r_{i \bar{j}}^{2}\right) \\
& \geq s-g^{i \bar{j}} g_{i \bar{j}} \\
& \geq 0 .
\end{aligned}
$$

Here we have used the Hessian comparison theorem on the distance functions proved in $[\mathbf{L W}]$ (see also $[\mathbf{C N}]$ Corollary 1.1).

q.e.d.

The following is a localized version of part (ii) of Theorem 3.3.

Proposition 5.2. Let

$$
E_{\mathcal{V}, x_{0}, t_{0}, t_{1}}(t)=\int_{\mathcal{V}} \varphi_{\left(x_{0}, t_{1}\right), \rho}(y, t) \hat{H}_{\left(x_{0}, t_{0}\right), \mathcal{V}}(y, t) d A_{\mathcal{V}}
$$


When in the right context we also briefly denote it by $E_{\mathcal{V}}$, then

$$
\frac{d}{d t} E_{\mathcal{V}}(t) \leq-\int_{\mathcal{V}}\left|\nabla^{\perp} \log \hat{H}_{\left(x_{0}, t_{0}\right), \mathcal{V}}\right|^{2} \varphi_{\left(x_{0}, t_{1}\right), \rho} \hat{H}_{\left(x_{0}, t_{0}\right), \mathcal{V}} d A_{\mathcal{V}}
$$

Proof. The computation (3.8) implies that

$$
\left(\frac{\partial}{\partial t}+\Delta \mathcal{V}\right) \hat{H}_{\left(x_{0}, t_{0}\right), \mathcal{V}} \leq-\left|\nabla^{\perp} \log \hat{H}_{\left(x_{0}, t_{0}\right), \mathcal{V}}\right|^{2} \hat{H}_{\left(x_{0}, t_{0}\right), \mathcal{V}}
$$

By Lemma 5.1 we have that

$$
\begin{aligned}
\frac{d}{d t} E_{\mathcal{V}}(t)= & \int_{\mathcal{V}}\left(\frac{\partial}{\partial t} \varphi_{\left(x_{0}, t_{1}\right), \rho}\right) \hat{H}_{\left(x_{0}, t_{0}\right), \mathcal{V}}+\varphi_{\left(x_{0}, t_{1}\right), \rho} \frac{\partial}{\partial t} \hat{H}_{\left(x_{0}, t_{0}\right), \mathcal{V}} d A_{\mathcal{V}} \\
= & \int_{\mathcal{V}}\left(\left(\frac{\partial}{\partial t}-\Delta_{\mathcal{V}}\right) \varphi_{\left(x_{0}, t_{1}\right), \rho}\right) \hat{H}_{\left(x_{0}, t_{0}\right), \mathcal{V}} d A_{\mathcal{V}} \\
& +\int_{\mathcal{V}} \varphi_{\left(x_{0}, t_{1}\right), \rho}\left(\left(\frac{\partial}{\partial t}+\Delta_{\mathcal{V}}\right) \hat{H}_{\left(x_{0}, t_{0}\right), \mathcal{V}}\right) d A_{\mathcal{V}} \\
& +\int_{\mathcal{V}}\left(\Delta_{\mathcal{V}} \varphi_{\left(x_{0}, t_{1}\right), \rho}\right) \hat{H}_{\left(x_{0}, t_{0}\right), \mathcal{V}}-\varphi_{\left(x_{0}, t_{1}\right), \rho}\left(\Delta_{\mathcal{V}} \hat{H}_{\left(x_{0}, t_{0}\right), \mathcal{V}}\right) d A_{\mathcal{V}} \\
\leq & -\int_{\mathcal{V}}\left|\nabla^{\perp} \log \hat{H}_{\left(x_{0}, t_{0}\right), \mathcal{V}}\right|^{2} \varphi_{\left(x_{0}, t_{1}\right), \rho} \hat{H}_{\left(x_{0}, t_{0}\right), \mathcal{V}} d A_{\mathcal{V}} .
\end{aligned}
$$

Here we have used the observation that

$$
\int_{\partial\left(\mathcal{V} \cap B_{x_{0}}\left(\sqrt{\rho^{2}+s\left(t_{0}-t\right)}\right)\right)} \hat{H}_{\left(x_{0}, t_{0}\right), \mathcal{V}}\left\langle\nabla_{\mathcal{V}} \varphi_{\left(x_{0}, t_{1}\right), \rho}, \nu\right\rangle d S \leq 0
$$

where $\nu$ is the unit out-normal of $\partial\left(\mathcal{V} \cap B_{x_{0}}\left(\sqrt{\rho^{2}+s\left(t_{1}-t\right)}\right)\right)$ in $\mathcal{V}$ and $d S$ is the area integral of $\partial\left(\mathcal{V} \cap B_{x_{0}}\left(\sqrt{\rho^{2}+s\left(t_{1}-t\right)}\right)\right)$.

In the above proof we have pretended that $\varphi_{\left(x_{0}, t_{0}\right), \rho}(y, t)$ is a smooth function with differential inequality (5.2). But in general it is only a Lipschitz function satisfying (5.2) in the sense of distribution. To make the argument rigorous we can introduce a cut-off function as in [E1]. For any $\epsilon>0$, let $\zeta_{\epsilon}(w) \in C^{1}(\mathbb{R})$ be a function satisfying $0 \leq \zeta_{\epsilon} \leq 1$, $\zeta_{\epsilon}(w)=1$ if $w \geq-n \log (1-\epsilon), \zeta_{\epsilon}(w)=0$ if $w \leq 0$ and $\left|\zeta_{\epsilon}^{\prime}\right| \leq \frac{2}{-n \log (1-\epsilon)}$. Hence $\left|\zeta_{\epsilon}^{\prime}(w)\right| w \leq 2$ on $[0,-n \log (1-\epsilon)]$ and $\zeta_{\epsilon}^{\prime}(w)=0$ for all other $w$. We may also assume that $\zeta_{\epsilon}^{\prime} \geq 0$. Let $\eta_{\epsilon}=\zeta_{\epsilon}\left(\varphi_{\left(x_{0}, t_{1}\right), \rho}\right)$. From the construction of $\zeta_{\epsilon}$ we have that $\left|\zeta_{\epsilon}^{\prime}\left(\varphi_{\left(x_{0}, t_{1}\right), \rho}\right)\right| \varphi_{\left(x_{0}, t_{1}\right), \rho} \leq 2$. It then is easy to see that

$$
\int_{\mathcal{V}} \varphi_{\left(x_{0}, t_{1}\right), \rho} \hat{H}_{\left(x_{0}, t_{0}\right), \mathcal{V}} \frac{\partial \eta_{\epsilon}}{\partial t} \rightarrow 0
$$

as $\epsilon \rightarrow 0$. Similarly, as $\epsilon \rightarrow 0$,

$$
\int_{\mathcal{V}} \varphi_{\left(x_{0}, t_{1}\right), \rho}\left|\nabla \hat{H}_{\left(x_{0}, t_{0}\right), \mathcal{V}}\right|\left|\nabla \eta_{\epsilon}\right| \rightarrow 0 .
$$

Now let

$$
E_{\mathcal{V}, \epsilon}(t)=\int_{M} \varphi_{\left(x_{0}, t_{1}\right), \rho} \hat{H}_{\left(x_{0}, t_{0}\right), \mathcal{V}} \eta_{\epsilon} d A_{\mathcal{V}}
$$


Then

$$
\begin{aligned}
& \frac{d}{d t} E_{\mathcal{V}, \epsilon}(t) \\
&=\int_{\mathcal{V}}\left(\frac{\partial}{\partial t} \varphi_{\left(x_{0}, t_{1}\right), \rho}\right) \hat{H}_{\left(x_{0}, t_{0}\right), \mathcal{V}} \eta_{\epsilon} \\
& \quad+\varphi_{\left(x_{0}, t_{1}\right), \rho} \eta_{\epsilon}\left(\frac{\partial}{\partial t} \hat{H}_{\left(x_{0}, t_{0}\right), \mathcal{V}}\right) d A_{\mathcal{V}}+\int_{\mathcal{V}} \varphi_{\left(x_{0}, t_{1}\right), \rho} \hat{H}_{\left(x_{0}, t_{0}\right), \mathcal{V}} \frac{\partial \eta_{\epsilon}}{\partial t} d A_{\mathcal{V}} \\
&=\int_{\mathcal{V}}\left(\frac{\partial}{\partial t} \varphi_{\left(x_{0}, t_{1}\right), \rho}\right) \eta_{\epsilon} \hat{H}_{\left(x_{0}, t_{0}\right), \mathcal{V}}+\left\langle\nabla_{\mathcal{V}} \varphi_{\left(x_{0}, t_{1}\right), \rho}, \nabla_{\mathcal{V}}\left(\eta_{\epsilon} \hat{H}_{\left(x_{0}, t_{0}\right), \mathcal{V}}\right)\right\rangle \\
&+\int_{\mathcal{V}} \varphi_{\left(x_{0}, t_{1}\right), \rho} \eta_{\epsilon}\left(\left(\frac{\partial}{\partial t}+\Delta \mathcal{V}\right) \hat{H}_{\left(x_{0}, t_{0}\right), \mathcal{V}}\right) \\
&-\int_{\mathcal{V}}\left\langle\nabla_{\mathcal{V}} \varphi_{\left(x_{0}, t_{1}\right), \rho}, \nabla_{\mathcal{V}} \eta_{\epsilon}\right\rangle \hat{H}_{\left(x_{0}, t_{0}\right), \mathcal{V}}-\left\langle\nabla_{\mathcal{V}} \varphi_{\left(x_{0}, t_{1}\right), \rho}, \nabla_{\mathcal{V}} \hat{H}_{\left(x_{0}, t_{0}\right), \mathcal{V}}\right\rangle \eta_{\epsilon} \\
&-\int_{\mathcal{V}} \varphi_{\left(x_{0}, t_{1}\right), \rho} \eta_{\epsilon}\left(\Delta \mathcal{V} \hat{H}_{\left.\left(x_{0}, t_{0}\right), \mathcal{V}\right)}\right)+\varphi_{\left(x_{0}, t_{1}\right), \rho} \hat{H}_{\left(x_{0}, t_{0}\right), \mathcal{V}} \frac{\partial \eta_{\epsilon}}{\partial t} \\
& \leq-\int_{\mathcal{V}}\left|\nabla^{\perp} \log \hat{H}_{\left(x_{0}, t_{0}\right), \mathcal{V}}\right|^{2} \varphi_{\left(x_{0}, t_{1}\right), \rho} \hat{H}_{\left(x_{0}, t_{0}\right), \mathcal{V}} d A_{\mathcal{V}} \\
&-\int_{\mathcal{V}}\left\langle\nabla_{\mathcal{V}} \varphi_{\left(x_{0}, t_{1}\right), \rho}, \nabla_{\mathcal{V}} \eta_{\epsilon}\right\rangle \hat{H}_{\left(x_{0}, t_{0}\right), \mathcal{V}}+\left\langle\nabla_{\mathcal{V}} \eta_{\epsilon}, \nabla_{\mathcal{V}} \hat{H}_{\left(x_{0}, t_{0}\right), \mathcal{V}}\right\rangle \varphi_{\left(x_{0}, t_{1}\right), \rho} \\
&+\int_{\mathcal{V}} \varphi_{\left(x_{0}, t_{1}\right), \rho} \hat{H}_{\left(x_{0}, t_{0}\right), \mathcal{V}} \frac{\partial \eta_{\epsilon}}{\partial t} .
\end{aligned}
$$

Observing that

$$
-\left\langle\nabla \mathcal{V} \varphi_{\left(x_{0}, t_{1}\right), \rho}, \nabla \mathcal{V} \eta_{\epsilon}\right\rangle \hat{H}_{\left(x_{0}, t_{0}\right), \mathcal{V}}=-\left|\nabla \varphi_{\left(x_{0}, t_{1}\right), \rho}\right|^{2} \zeta^{\prime}\left(\varphi_{\left(x_{0}, t_{1}\right), \rho}\right) \leq 0
$$

and the last two terms, in the above estimate of $\frac{d}{d t} E_{\mathcal{V}, \epsilon}(t)$, tend to zero as $\epsilon \rightarrow 0$, we have the claimed monotonicity by taking $\epsilon \rightarrow 0$. $\quad$ q.e.d.

We denote the $2 s$-dimensional Hausdorff measure of set $\mathcal{V} \cap B_{x_{0}}(\rho)$ by $\mathcal{A}_{\mathcal{V}, x_{0}}(\rho)$. As a consequence we have the following elliptic 'monotonicity principle' (can be viewed a Bishop-Lelong lemma on manifolds).

Corollary 5.3 (Monotonicity principle). Let $\delta(s)=\frac{1}{\sqrt{2+4 s}}$. There exists $C=C(m, s)$ such that for any $\rho^{\prime} \in(0, \delta(s) \rho)$

$$
\frac{\mathcal{A}_{\mathcal{V}, x_{0}}\left(\rho^{\prime}\right)\left(\rho^{\prime}\right)^{2(m-s)}}{V_{x_{0}}\left(\rho^{\prime}\right)} \leq C(m, s) \frac{\mathcal{A}_{\mathcal{V}, x_{0}}(\rho) \rho^{2(m-s)}}{V_{x_{0}}(\rho)} .
$$

Proof. The proof follows essentially the argument of Proposition 3.5 in [E2], applying Proposition 5.2 with $t_{1}=t_{0}-\delta^{2}(s) \rho^{2}$ and $t_{0}$ replaced 
by $t_{0}+\rho^{\prime 2}$. Using the heat kernel upper bound of Li-Yau we have that

$$
\begin{aligned}
& \hat{H}_{\left(x_{0}, t_{0}+\rho^{\prime 2}\right), \mathcal{V}}\left(y, t_{0}-\delta^{2}(s) \rho^{2}\right) \\
& \leq\left(\pi\left(\delta^{2}(s) \rho^{2}+\rho^{\prime 2}\right)\right)^{m-s} \frac{C(m)}{V_{x_{0}}\left(\sqrt{{\rho^{\prime}}^{2}+\delta^{2}(s) \rho^{2}}\right)} \\
& \leq C(m, s) \frac{\left(\pi \rho^{2}\right)^{m-s}}{V_{x_{0}}(\delta(s) \rho)} \\
& \leq C(m, s) \frac{\left(\pi \rho^{2}\right)^{m-s}}{V_{x_{0}}(\rho)} .
\end{aligned}
$$

Here we have used the Bishop volume comparison in the last inequality. Notice that $\varphi_{\left(x_{0}, t_{1}\right), \rho}\left(x, t_{0}-\delta^{2}(s) \rho^{2}\right) \leq 1$ and supported inside $B_{x_{o}}(\rho)$. Hence (5.7) implies that

$$
E_{\mathcal{V}}\left(t_{0}-\delta^{2}(s) \rho^{2}\right) \leq C(m, s) \frac{\mathcal{A}_{\mathcal{V}, x_{0}}(\rho) \rho^{2(m-s)}}{V_{x_{0}}(\rho)} .
$$

By Proposition 5.2 we know that

$$
E_{\mathcal{V}}\left(t_{0}-\delta^{2}(s) \rho^{2}\right) \geq E_{\mathcal{V}}\left(t_{0}-\rho^{\prime 2}\right) .
$$

On the other hand, by Li-Yau's heat kernel lower bound we also have that, for all $y \in B_{x_{0}}\left(\rho^{\prime}\right)$,

$$
\begin{aligned}
& \hat{H}_{\left(x_{0}, t_{0}+\rho^{\prime 2}\right), \mathcal{V}}\left(y, t_{0}-\rho^{\prime 2}\right) \\
& \geq\left(\pi\left(2 \rho^{\prime 2}\right)\right)^{m-s} \frac{C(m)}{V_{x_{0}}\left(\sqrt{2} \rho^{\prime}\right)} \exp \left(-\frac{r^{2}\left(x_{0}, y\right)}{6 \rho^{\prime 2}}\right) \\
& \geq C(m, s) \frac{\left(\pi \rho^{\prime 2}\right)^{m-s}}{V_{x_{0}}\left(\rho^{\prime}\right)} .
\end{aligned}
$$

Again we have used the Bishop volume comparison theorem. Notice that for $y \in B_{x_{0}}\left(\rho^{\prime}\right)$

$$
\begin{aligned}
\varphi_{\left(x_{0}, t_{1}\right), \rho}\left(y, t_{0}-\rho^{\prime 2}\right) & \geq 1-\frac{\rho^{\prime 2}+s\left(\delta^{2}(s) \rho^{2}+{\rho^{\prime}}^{2}\right)}{\rho^{2}} \\
& \geq 1-\delta^{2}(s)(1+2 s) \\
& =\frac{1}{2} .
\end{aligned}
$$

Combining (5.10), (5.11) we have that

$$
E_{\mathcal{V}}\left(t_{0}-\rho^{\prime 2}\right) \geq C(m, s) \frac{\rho^{\prime 2(m-s)} \mathcal{A}_{\mathcal{V}, x_{0}}\left(\rho^{\prime}\right)}{V_{x_{0}}\left(\rho^{\prime}\right)} .
$$

Combining (5.8), (5.9) and (5.12) we complete the proof.

q.e.d. 
Remark 5.4. In Proposition 3.1.1 of [M1], a (considerably weaker) comparison on the relative volumes (in the spirit of Corollary 5.3) was first established for the zero divisors of holomorphic functions of polynomial growth, under further assumptions on $M$ being of maximum volume growth and of quadratic curvature decay, using a very different method.

The following consequence of Corollary 5.3 is somewhat surprising. The result sharpens the Bishop-Gromov volume comparison theorem in the presence of compact subvarieties.

Corollary 5.5. Let $M^{m}$ be a complete Kähler manifold with nonnegative holomorphic bisectional curvature. Suppose that $M$ contains a compact subvariety $\mathcal{V}$ of complex dimension $s$. Then there exists $C=C(m, s)>0$ such that for $\delta(s) \rho \geq \rho^{\prime} \gg 1$,

$$
\frac{V_{x_{0}}(\rho)}{V_{x_{0}}\left(\rho^{\prime}\right)} \leq C\left(\frac{\rho}{\rho^{\prime}}\right)^{2(m-s)}
$$

In particular,

$$
\limsup _{\rho \rightarrow \infty} \frac{V_{x_{0}}(\rho)}{\rho^{2(m-s)}}<\infty .
$$

By application of the above 'monotonicity principle' we can have another proof of Theorem 3.1 of [N4]. In fact the new proof gives a characterization of divisors defined by holomorphic functions of polynomial growth (also called 'polynomial functions' according to the notation in [W2]).

Theorem 5.6. Let $M$ be a complete Kähler manifold with nonnegative bisectional curvature. Let $\mathcal{V}$ be an analytic divisor of $M$. Define the Lelong number (elliptic) at infinity of $\mathcal{V}$ by

$$
\nu_{\infty}(\mathcal{V})=\sup _{x_{0} \in M} \limsup _{\rho \rightarrow \infty} \frac{\pi \rho^{2} \mathcal{A}_{\mathcal{V}, x_{0}}(\rho)}{V_{x_{0}}(\rho)} .
$$

Assume further that $H^{1}\left(M, \mathcal{O}^{*}\right)=0$. Then $\mathcal{V}$ is defined by a polynomial function if and only if $\nu_{\infty}(\mathcal{V})<\infty$. Moreover, if $\mathcal{V}=Z(f)$ for some $f \in P_{d}(M)$ (the space of holomorphic functions of polynomial growth with degree at most $d$ ) then there exists a $C(m)$ such that for any $x_{0} \in$ $M$, the Lelong number $\nu\left(x_{0}, \mathcal{V}\right)$ at $x_{0}$ is bounded by

$$
\nu\left(x_{0}, \mathcal{V}\right) \leq C(m) \nu_{\infty}(\mathcal{V})
$$

and

$$
\nu_{\infty}(\mathcal{V}) \leq C(m) d .
$$

Proof. Notice that (5.14) follows from Corollary 5.3 directly. First assume that $\mathcal{V}$ is the zero divisor of a polynomial function $f\left(\in P_{d}(M)\right.$ for some $d)$. We shall show (5.15), which certainly implies that $\nu_{\infty}(\mathcal{V})<$ 
$\infty$. We follow the notation in Section 3 of $[\mathbf{N 4}]$ (also Section 3 of this paper). Let $v(x, t)=\int_{M} H(x, y, t) \Delta \log |f|^{2} d v_{y}$ and $w(x, t)=\frac{\partial}{\partial t} v(x, t)$. By Corollary 3.5 we know that

$$
t w(x, t)=(\pi t) \int_{\mathcal{V}} H(x, y, t) d A_{\mathcal{V}}
$$

By Li-Yau's lower bound estimate on heat kernel we have that

$$
t w(x, t) \geq C(m) \frac{\pi t \mathcal{A}_{\mathcal{V}, x}(\sqrt{t})}{V_{x}(\sqrt{t})} .
$$

Then (5.15) follows from (3.13) of [N4].

Now we assume that $\nu_{\infty}(\mathcal{V})<\infty$ and we prove that $\mathcal{V}$ is the divisor of a polynomial function. First, by the solution to Cousin problem II (directly from the vanishing of the cohomology $H^{1}\left(M, \mathcal{O}^{*}\right)$ ) we know that there exists a holomorphic function $f$ such that $Z(f)=\mathcal{V}$. First we apply Theorem 3.1 of [N1], a 'moment type estimate' to estimate $t w(x, t)$ from above. Note that $w(x, t)=\int_{\mathcal{V}} H(x, y, t) d A_{\mathcal{V}}(y)$ is welldefined due to the assumption that $\nu_{\infty}(\mathcal{V})<\infty$. By Corollary 3.5 we know that

$$
w(x, t)=\int_{M} H(x, y, t) \Delta \log |f|^{2} d \mu(y) .
$$

Applying the 'moment type estimate', Theorem 3.1 of [N1], we have that

$$
t w(x, t) \leq C(m) \nu_{\infty}(\mathcal{V}) .
$$

Now we define $v(x, t)=\int_{0}^{t} w(x, \tau) d \tau+\log |f|^{2}(x)$. Then (5.16) implies that $v(x, t) \leq C(m) \nu_{\infty}(\mathcal{V}) \log (t+1)+\log |f|^{2}(x)$ for $t \gg 1$. (Here $x$ is chosen so that $x \in M \backslash \mathcal{V}$.) One can also check that $v(x, t)$ is a solution to the heat equation $\left(\frac{\partial}{\partial t}-\Delta\right) v(x, t)=0$ with $v(x, 0)=\log |f|^{2}(x)$. Now again we apply the 'moment type estimate', Theorem 3.1 of [N1], and we have that

$$
f_{B_{x}(r)} \log |f|^{2}(y) d \mu(y) \leq C(m, x) \nu_{\infty}(\mathcal{V}) \log (r+1) .
$$

Now applying the mean-value inequality of Li-Schoen $[\mathbf{L S}]$ to the subharmonic function $\log |f|^{2}$ we conclude that $f$ is of polynomial growth.

q.e.d.

\section{Remark 5.7.}

(1) From the proof it is easy to see that one only needs $\nu\left(\mathcal{V}, x_{0}\right)<\infty$ for some $x_{0}$ to conclude that the defining function $f$ is a 'polynomial function'. Here

$$
\nu\left(\mathcal{V}, x_{0}\right):=\limsup _{r \rightarrow \infty} \frac{\pi \rho^{2} \mathcal{A}_{\mathcal{V}, x_{0}}(\rho)}{V_{x_{0}}(\rho)} .
$$


We do not have to worry that in the proof $x$ may be on $\mathcal{V}$ since the finiteness of $\nu\left(\mathcal{V}, x_{0}\right)$ implies the finiteness of $\nu(\mathcal{V}, x)$ for any $x \in M$.

(2) In $[\mathbf{S t}]$ (see also $[\mathbf{R u}]$ for the codimension one case), Stoll proved that an analytic divisor $\mathcal{V}$ in $M=\mathbb{C}^{m}$ is algebraic if and only if $\nu_{\infty}(\mathcal{V})<\infty$. The result was later generalized to the case $M$ being an affine algebraic variety in $[\mathbf{G K}]$. In fact, in $[\mathbf{S t}]$ the result was proved for any analytic sets of $\mathbb{C}^{m}$. It is desirable to generalize our result to the high codimension case.

(3) The assumption on vanishing of the cohomology in the above theorem is satisfied, for example, when $M$ is Stein (cf. Theorem 5.5.2 of $[\mathbf{H o}])$.

In [N4], the author developed a parabolic approach to compare the vanishing order of a holomorphic function, at any fixed point, with the growth order at infinity. The method is sharp and effective. It turns out that the parabolic method there is also related to the Nevanlinna theory for several complex variables. In order to illustrate this connection we need to recall some basic notations from the Nevanlinna theory (cf. $[\mathbf{G}, \mathbf{W} \mathbf{1}])$.

We say that a function $s: \mathbb{R}_{+} \rightarrow \mathbb{R}_{+}$has finite order if

$$
\operatorname{Ord}(s):=\limsup _{r \rightarrow \infty} \frac{\log s(r)}{\log r}<\infty .
$$

For a $f \in \mathcal{O}(M)$, the space of holomorphic functions, we define the order of $f$ in sense of Hadamard by

$$
\operatorname{Ord}_{H}(f)=\operatorname{Ord}(\log (A(r)))
$$

where $A(r)=\sup _{x \in B_{o}(r)}|f|(x)$ with $o \in M$ being a fixed point. Following $[\mathbf{G}, \mathbf{S t}]$ for any analytic subvariety of complex dimension $s$ we define

$$
n_{\mathcal{V}}\left(x_{0}, r\right)=\frac{\mathcal{A}_{x_{0}}(r)\left(\pi r^{2}\right)^{m-s}}{V_{x_{0}}(r)}
$$

and

$$
N_{\mathcal{V}}\left(x_{0}, r\right)=\int_{0}^{r}\left(n_{\mathcal{V}}\left(x_{0}, \tau\right)-n_{\mathcal{V}}\left(x_{0}, 0\right)\right) \frac{d \tau}{\tau}+n_{\mathcal{V}}\left(x_{0}, 0\right) \log r
$$

$N_{\mathcal{V}}\left(x_{0}, r\right)\left(n_{\mathcal{V}}\left(x_{0}, r\right)\right)$ is called the counting function since for $m=1$ and $\mathcal{V}$ being the zeros of a holomorphic function, it simply counts the number of zeros in $B\left(x_{0}, r\right)$. If $x_{0}$ does not lie in $\mathcal{V}$ one has $N_{\mathcal{V}}\left(x_{0}, r\right)=$ $\int_{0}^{r} n_{\mathcal{V}}\left(x_{0}, \tau\right) \frac{d \tau}{\tau}$. One can view the estimate (5.15) as bounding the counting function by the growth order, a Nevanlinna type inequality. The following result is a further generalization of (5.15), whose correspondence in the Euclidean space is known as the transcendental Bézout estimate. 
Corollary 5.8. Let $M$ be a complete noncompact Kähler manifold with non-negative Ricci curvature. Let $f \in \mathcal{O}(M)$ be a holomorphic function of finite order. Let $Z(f)$ be the zero divisor of $f$. Then

$$
\operatorname{Ord}\left(N_{Z}\left(x_{0}, r\right)\right) \leq \operatorname{Ord}_{H}(f) .
$$

Proof. The proof follows the same line of argument as the proof of Theorem 5.6. We leave it to the interested readers.

q.e.d.

We found the connection between the parabolic equations (as well as the related differential Harnack inequality) and the Nevanlinna theory quite interesting.

\section{The heat kernel and the reduced volumes}

In this section we consider the fundamental solution $H\left(y, t ; x, t_{0}\right)$ to the forward conjugate heat equation for a family of metrics deformed by Kähler-Ricci/Ricci flow (2.1). We shall prove a sharp lower bound on $H\left(y, t ; x, t_{0}\right)$. In [N5] we derived an estimate for the fundamental solution of the time-dependent heat equation itself (instead of $(2.2))$. The estimate there is only valid for short time intervals. In $[\mathbf{G u}]$, a rough lower bound was obtained through the earlier mentioned Harnack inequality, Theorem 2.7. Notice that the result in $[\mathbf{G u}]$ is not sharp and the result in [N5] is only sharp in the exponents. In this section we show a sharp lower bound for the fundamental solution to the forward conjugate heat equation in the case that $M$ is either a complete Riemannian bounded nonnegative curvature operator or a complete Kähler manifold with bounded nonnegative bisectional curvature. For the sake of simplicity we only state the result for the Kähler-Ricci flow and leave the Riemannian analogue to the interested readers. Before we state our result we need to recall some notations and computations from [FIN] (which follow closely the computation in $[\mathbf{P}]$ ). For simplicity we assume that $t_{0}=0$.

Let $g(t)$ be a complete solution to Kähler-Ricci flow on $M^{m} \times[0, T]$ (where $m=\operatorname{dim}_{\mathbb{C}}(M)$ and $n=2 m$ ). Fix $x_{0}$ and let $\gamma$ be a path $(x(\eta), \eta)$ joining $\left(x_{0}, 0\right)$ to $(y, t)$. Following $[\mathbf{P}]$ (see also $[\mathbf{L Y}, \mathbf{F I N}]$ ) we define

$$
\mathcal{L}_{+}(\gamma)=\int_{0}^{t} \sqrt{\eta}\left(\mathcal{R}+4\left|\gamma^{\prime}(\eta)\right|^{2}\right) d \eta
$$

Let $X=\gamma^{\prime}(t)=\frac{d z^{\alpha}(t)}{d t} \frac{\partial}{\partial z^{\alpha}}$ and let $Y$ be a variational vector field along $\gamma$. Here $\left|\gamma^{\prime}(t)\right|^{2}=g_{\alpha \bar{\beta}} \frac{d z^{\alpha}(t)}{d t} \frac{d z^{\bar{\beta}}(t)}{d t}$. Using $\mathcal{L}_{+}$as energy we can define the $\mathcal{L}_{+}$-geodesics and we denote $L_{+}(y, t)$ to be the length of the shortest geodesic jointing $\left(x_{0}, 0\right)$ to $(y, t)$. We also define

$$
\ell_{+}\left(y, t ; x_{0}, 0\right):=\frac{1}{2 \sqrt{t}} L_{+}(y, t) .
$$


Following the first and second variation calculation of $[\mathbf{P}]$ (see also [FIN]) we have that

$$
\begin{aligned}
\left|\nabla \ell_{+}\right|^{2} & =-\mathcal{R}+\frac{\ell_{+}}{t}+\frac{K}{t^{3 / 2}}, \\
\frac{\partial \ell_{+}}{\partial t} & =\mathcal{R}-\frac{K}{2 t^{3 / 2}}-\frac{\ell_{+}}{t}, \\
\Delta \ell_{+} & \leq \mathcal{R}+\frac{n}{2 t}-\frac{K}{2 t^{3 / 2}} .
\end{aligned}
$$

Here

$$
K:=\int_{0}^{t} \eta^{3 / 2} H(X) d \eta
$$

where $H(X):=\partial \mathcal{R} / \partial t+2\langle\nabla \mathcal{R}, X\rangle+2\langle X, \nabla \mathcal{R}\rangle+4 \operatorname{Ric}(X, X)+\mathcal{R} / t$ is exactly the traced LYH differential Harnack expression in $[\mathbf{C 1}]$ applying to the $(1,0)$ vector field $2 X$.

Theorem 6.1. Let $\left(M^{m}, g(t)\right)$ be a complete solution to a KählerRicci (Ricci) flow with bounded nonnegative bisectional curvature (curvature operator $)$. Let $H\left(y, t ; x_{0}, 0\right)$ be the fundamental solution to the forward conjugate heat equation centered at $\left(x_{0}, 0\right)$. Then

$$
\tilde{u}(y, t):=\frac{1}{(\pi t)^{m}} \exp \left(-\ell_{+}\left(y, t ; x_{0}, 0\right)\right)
$$

satisfies

$$
\left(\frac{\partial}{\partial t}-\Delta-\mathcal{R}\right) \tilde{u}(y, t) \leq 0
$$

In particular,

$$
\tilde{u}\left(y, t ; x_{0}, 0\right) \leq H\left(y, t ; x_{0}, 0\right)
$$

and

$$
\tilde{\theta}_{+}^{\left(x_{0}, 0\right)}(t):=\int_{M} \tilde{u}(y, t) d \mu_{t}(y)
$$

is monotone decreasing. Moreover, the equality in (6.5), or (6.6) implies that $M$ is a gradient expanding soliton.

Proof. First (6.2)-(6.4) implies that

$$
\begin{aligned}
& \left(\frac{\partial}{\partial t}-\Delta-\mathcal{R}\right)\left(\frac{1}{(\pi t)^{m}} \exp \left(-\ell_{+}(y, t)\right)\right) \\
& =-\frac{K}{t^{\frac{3}{2}}}\left(\frac{1}{(\pi t)^{m}} \exp \left(-\ell_{+}(y, t)\right)\right) \leq 0 .
\end{aligned}
$$

Here we have used the fact that $K \geq 0$ under the assumption that $M$ has bounded non-negative bisectional curvature. Also, if the equality holds it implies that $K \equiv 0$. This further implies that $M$ is an expanding soliton from the computation in $[\mathbf{F I N}]$. In order to prove (6.6) one just needs to apply the maximum principle (cf. $[\mathbf{N T 2}, \mathbf{N 5}]$ ) and notice that 
$\lim _{t \rightarrow 0} \frac{1}{(\pi t)^{m}} \exp \left(-\ell_{+}(y, t)\right)=\delta_{x_{0}}(y)$. The equality case follows from the analysis on the equality case in $[\mathbf{F I N}]$.

q.e.d.

Remark 6.2. In $[\mathbf{C Y}]$, Cheeger and Yau proved that for complete manifolds with nonnegative Ricci curvature, the heat kernel $H(x, y, t)$ has the lower bound estimate

$$
\bar{H}(r(x, y), t):=\frac{1}{(4 \pi t)^{\frac{n}{2}}} \exp \left(-\frac{r^{2}(x, y)}{4 t}\right) \leq H(x, y, t)
$$

by showing that the transplant of the Euclidean heat kernel $\bar{H}(r(x, y), t)$ is a sub-solution to the heat equation. (Here $r(x, y)$ is the distance function as before.) Recall that in $[\mathbf{P}]$, Perelman first discovered that there is a similar result for the backward Ricci flow, even without any curvature sign assumptions (this is the astonishing part of Perelman's work). Namely he proved, for $\frac{\partial}{\partial \tau} g_{i j}=2 R_{i j}$, that one can define the reduced distance $\ell(y, \tau)$ (formally by the same expression as $\ell_{+}$) and that

$$
\left(\frac{\partial}{\partial \tau}-\Delta+\mathcal{R}\right) \bar{u}(y, \tau) \leq 0
$$

where

$$
\bar{u}(y, \tau):=\frac{1}{(4 \pi \tau)^{\frac{n}{2}}} \exp (-\ell(y, \tau)) .
$$

Perelman further showed (in Corollary 9.5 of $[\mathbf{P}]$ ) that $\bar{u}$ gives a lower bound for the fundamental solution to the backward conjugate heat equation $\left(\frac{\partial}{\partial \tau}-\Delta+\mathcal{R}\right) u(y, \tau)=0$. One should also refer to Theorem 4.3 in $[\mathbf{L Y}]$ for a precedence of Perelman's second variation computation in $[\mathbf{P}]$ Section 7 . Thus both Perelman's monotonicity of the reduced volume and Theorem 6.1 above can be viewed as a nonlinear analogue of the earlier work of Cheeger-Yau in $[\mathbf{C Y}]$ and Li-Yau in $[\mathbf{L Y}]$.

Tracing the computation of $[\mathbf{F I N}]$ we also have the following estimates for $\tilde{u}(x, t)$.

Proposition 6.3. Assume that $(M, g(t))$ is a Kähler-Ricci flow with bounded nonnegative bisectional curvature on $M \times[0, T)$. Let $\tilde{u}(x, t)$ be as in Theorem 5.1. Then

$$
\log (\tilde{u})_{\alpha \bar{\beta}}+R_{\alpha \bar{\beta}}+\frac{1}{t} g_{\alpha \bar{\beta}} \geq 0 .
$$

The equality holds if and only if $(M, g(t))$ is an expanding Kähler-Ricci solution.

Notice that (6.8) is equivalent to the estimate $(2.3)$ on $u(x, t)$, any positive solution to the forward conjugate heat equation. On the other hand, $\tilde{u}(x, t)$ is only a sub-solution to the forward conjugate heat equation. 
Remark 6.4. In Corollary 2.1 of [FIN], we showed that under the assumption of bounded nonnegative curvature operator there exists exactly the same estimate as (6.8) (simply without the bars). This suggests that one may have an estimate similar to Theorem 2.2 for the Riemannian case on positive solution $u$ of the forward conjugate heat equation. Indeed, this can be shown if we can prove, under the assumption of $M$ having bounded nonnegative curvature operator, that

$$
\begin{aligned}
Y_{i j}:= & \nabla_{i} \nabla_{j} \mathcal{R}-2 \nabla_{k} R_{i j} \nabla_{k}(\log u)+2 R_{i k j l} \nabla_{k}(\log u) \nabla_{l}(\log u) \\
& +R_{i k} R_{j k}+\frac{1}{t} R_{i j} \geq 0 .
\end{aligned}
$$

This claim is based on the following computation. Note that $Y_{i j} \equiv 0$ on gradient expanding solitons and its trace is the same as the trace of Hamilton's matrix LYH expression in $[\mathbf{H 1}]$.

Lemma 6.5. Let

$$
\tilde{Z}_{i j}=R_{i j}+(\log u)_{i j}+\frac{1}{2 t} g_{i j} .
$$

Then

$$
\begin{aligned}
\left(\frac{\partial}{\partial t}-\Delta_{L}\right) \tilde{Z}_{i j}= & Y_{i j}+2 \nabla_{k} \tilde{Z}_{i j} \nabla_{k}(\log u) \\
& +\tilde{Z}_{i k}\left(\log (u)_{j k}-R_{j k}-\frac{1}{2 t} g_{j k}\right) \\
& +\left(\log (u)_{i k}-R_{i k}-\frac{1}{2 t} g_{i k}\right) \tilde{Z}_{j k}
\end{aligned}
$$

where $\Delta_{L} \eta_{i j}=\Delta \eta_{i j}+2 R_{i k j l} \eta_{k l}-R_{i k} \eta_{k j}-R_{i k} \eta_{i k}$ is the Lichnerowicz operator acting on the symmetric 2 -tensor $\eta_{i j}$.

The above computation was first carried out in $[\mathbf{C L N}]$ for the backward Ricci flow, with $Y_{i j}$ being replaced by Hamilton's matrix Harnack expression for shrinkers. Notice also that the matrix Harnack expression $Y_{i j}$ and Hamilton's expression for Ricci expanders are the same if the manifold is Kähler.

One can obtain some upper bound on the heat kernel $H(x, y, t, 0)$ using the Harnack inequality proved in Corollary 3.8 and the fact that $\int_{M} H(x, y, t, 0) d \mu_{t}=1$. But the result is not as satisfactory as for the fixed metric case of Li-Yau. Hence we shall leave this to a later investigation. On the other hand, the localization techniques of Ecker can be applied to the reduced volume for the Ricci expanders (defined in $[$ FIN]) to obtain the monotonicity of a localized reduced volume without assuming bisectional curvature (nor curvature operator) being nonnegative. Recall that in [FIN], the authors proved that, if $M$ is a 
closed manifold, the forward reduced volume

$$
\theta_{+}^{\left(x_{0}, 0\right)}(t):=\int_{M} \hat{u}(x, t) d \mu_{t}
$$

is monotone non-increasing, where $\hat{u}(x, t)=\frac{e^{\ell}(x, t)}{(\pi t)^{m}}$. (We call $\tilde{\theta}_{+}^{\left(x_{0}, 0\right)}$, defined in Theorem 6.1, the second forward reduced volume.) Here we define $\hat{u}$ for the Kähler case to be coherent with our previous discussions. This monotonicity has a severe restriction since the reduced volume $\theta_{+}^{\left(x_{0}, 0\right)}(t)$ is only meaningful when $M$ is compact. We shall show the monotonicity of a localized reduced volume, which is well-defined on complete manifolds, to compensate such restriction. In order to put the result in its general form we denote by $\ell_{+}^{\left(x_{0}, t_{0}\right)}$ the forward reduced distance with respect to $\left(x_{0}, t_{0}\right)$ if one replaces the reference point $\left(x_{0}, 0\right)$ with $\left(x_{0}, t_{0}\right)$. Correspondingly we denote by $\hat{u}^{\left(x_{0}, t_{0}\right)}$ and $\theta_{+}^{\left(x_{0}, t_{0}\right)}$, the reduced volume function and the reduced volume with respect to $\left(x_{0}, t_{0}\right)$, respectively. Let

$$
\bar{L}_{+}^{\left(x_{1}, t_{1}\right)}=\left(t-t_{1}\right) \ell_{+}^{\left(x_{1}, t_{1}\right)}
$$

and

$$
\varphi_{t_{2}, \rho}^{\left(x_{1}, t_{1}\right)}(x, t)=\left(1-\frac{\bar{L}_{+}^{\left(x_{1}, t_{1}\right)}(x, t)+m\left(t-t_{2}\right)}{\rho^{2}}\right)_{+} .
$$

Then (6.2)-(6.4) imply that

$$
\left(\frac{\partial}{\partial t}+\Delta-\mathcal{R}\right) \hat{u}^{\left(x_{0}, t_{0}\right)}(x, t) \leq 0
$$

and

$$
\left(\frac{\partial}{\partial t}-\Delta\right) \varphi_{t_{2}, \rho}^{\left(x_{1}, t_{1}\right)}(x, t) \leq 0
$$

Notice that if $\mathcal{R}$ is uniformly bounded from below, $\varphi_{t_{2}, \rho}^{\left(x_{1}, t_{1}\right)}(x, t)$ is compactly supported. We then have the following localized monotonicity of the reduced volume.

\section{Proposition 6.6.}

$$
\frac{d}{d t} \theta_{+, \varphi}^{\left(x_{0}, t_{0}\right)}(t) \leq 0
$$

where

$$
\theta_{+, \varphi}^{\left(x_{0}, t_{0}\right)}(t)=\int_{M} \varphi_{t_{2}, \rho}^{\left(x_{1}, t_{1}\right)}(x, t) \hat{u}^{\left(x_{0}, t_{0}\right)}(x, t) d \mu_{t} .
$$

It turns out that one can construct another sub-solution to the heat equation with compact support, similar to $\varphi_{t_{2}, \rho}^{\left(x_{1}, t_{1}\right)}(x, t)$ defined above, through Perelman's reduced distance $\ell^{\left(x_{1}, t_{1}\right)}(x, \tau)$, with $\tau=t_{1}-t$. (Note that $\ell^{\left(x_{1}, t_{1}\right)}\left(x, t_{1}-t\right)$ is only defined for $t \leq t_{1}$. Please refer to $[\mathbf{P}]$ 
Section 7 for the detailed discussions.) Here we use $\left(x_{1}, t_{1}\right)$ to specify the reference space-time point with respect to which the reduced distance is defined. Recall that from $[\mathbf{P}]$ Section $7, \bar{L}^{\left(x_{1}, t_{1}\right)}(x, t):=$ $\left(t_{1}-t\right) \ell^{\left(x_{1}, t_{1}\right)}\left(x, t_{1}-t\right)$ (again we do not have the factor 4 since we are in the Kähler setting) satisfies the differential inequality:

$$
\left(-\frac{\partial}{\partial t}+\Delta\right) \bar{L}^{\left(x_{1}, t_{1}\right)}(x, t) \leq m
$$

Then in the case $\mathcal{R}$ is bounded from below, we may define a compact supported function

$$
\psi_{t_{2}, \rho}^{\left(x_{1}, t_{1}\right)}(x, t):=\left(1-\frac{\bar{L}^{\left(x_{1}, t_{1}\right)}(x, t)+m\left(t-t_{2}\right)}{\rho^{2}}\right)_{+} .
$$

It is easy to see that

$$
\left(\frac{\partial}{\partial t}-\Delta\right) \psi_{t_{2}, \rho}^{\left(x_{1}, t_{1}\right)}(x, t) \leq 0
$$

This gives another localization on the forward reduced volume.

\section{Proposition 6.7.}

$$
\frac{d}{d t} \theta_{+, \psi}^{\left(x_{0}, t_{0}\right)}(t) \leq 0
$$

where

$$
\theta_{+, \varphi}^{\left(x_{0}, t_{0}\right)}(t)=\int_{M} \psi_{t_{2}, \rho}^{\left(x_{1}, t_{1}\right)}(x, t) \hat{u}^{\left(x_{0}, t_{0}\right)}(x, t) d \mu_{t} .
$$

The similar idea can also be applied to Perelman's entropy. Let $\left(x_{0}, t_{0}\right)$ be a fixed space-time point. Let $\tau=t_{0}-t$ and $u(x, \tau)$ be the fundamental solution of the backward conjugate heat equation $\frac{\partial}{\partial \tau}-\Delta+\mathcal{R}$ centered at $\left(x_{0}, t_{0}\right)$. Write $u=\frac{e^{-f}}{(\pi \tau)^{m}}$ and define

$$
v=\left(\tau\left(2 \Delta f-|\nabla f|^{2}+\mathcal{R}\right)+f-2 m\right) u .
$$

Then it was proved in $[\mathbf{P}]$ that

$$
\left(\frac{\partial}{\partial \tau}-\Delta+\mathcal{R}\right) v=-\tau\left(\left|R_{\alpha \bar{\beta}}+\nabla_{\alpha} \nabla_{\bar{\beta}} f-\frac{1}{\tau} g_{\alpha \bar{\beta}}\right|^{2}+\left|\nabla_{\alpha} \nabla_{\beta} f\right|^{2}\right) u
$$

and $v \leq 0$. Applying (6.12) or (6.15) we can have the following local monotonicity formulae.

\section{Proposition 6.8.}

$$
\begin{aligned}
& \frac{d}{d t}\left(\int_{M}-v \varphi_{t_{2}, \rho}^{\left(x_{1}, t_{1}\right)} d \mu_{t}\right) \\
& \leq-\int_{M} \tau\left(\left|R_{\alpha \bar{\beta}}+\nabla_{\alpha} \nabla_{\bar{\beta}} f-\frac{1}{\tau} g_{\alpha \bar{\beta}}\right|^{2}+\left|\nabla_{\alpha} \nabla_{\beta} f\right|^{2}\right) u \varphi_{t_{2}, \rho}^{\left(x_{1}, t_{1}\right)} d \mu_{t}
\end{aligned}
$$


and

$$
\begin{aligned}
\text { (6.20) } & \frac{d}{d t}\left(\int_{M}-v \psi_{t_{2}, \rho}^{\left(x_{1}, t_{1}\right)} d \mu_{t}\right) \\
\leq & -\int_{M} \tau\left(\left|R_{\alpha \bar{\beta}}+\nabla_{\alpha} \nabla_{\bar{\beta}} f-\frac{1}{\tau} g_{\alpha \bar{\beta}}\right|^{2}+\left|\nabla_{\alpha} \nabla_{\beta} f\right|^{2}\right) u \psi_{t_{2}, \rho}^{\left(x_{1}, t_{1}\right)} d \mu_{t} .
\end{aligned}
$$

Proof. The proof is just direct computations and integration by parts, using (6.12), (6.15) and (6.18).

q.e.d.

There is no difference for the Riemannian cases. The formulae are exactly the same except some factors caused by the different definitions of the Laplacian and gradient operators.

In the derivation of Propositions 6.6, 6.7 and 6.8 we have pretended that all functions involved are smooth. In general this may not be the case. For example, we only know that $\varphi_{t_{2}, \rho}^{\left(x_{1}, t_{1}\right)}$ and $\psi_{t_{2}, \rho}^{\left(x_{1}, t_{1}\right)}$ are Lipschitz and satisfy the claimed differential inequality in the sense of distributions (cf. [Ye]). However, we can follow the earlier proof of Proposition 5.2 to make the derivation rigorous. This approximation argument can also be found in Section 2 of [E1]. We leave the detailed rigorous proof to the interested readers.

We conclude this section by an application of Theorem 6.1 to the study of the large time behavior of Kähler-Ricci flow. Let $\left(M^{m}, g_{0}\right)$ be a complete Kähler manifold with bounded nonnegative holomorphic bisectional curvature. If we further assume that $\left(M, g_{0}\right)$ has maximum volume growth, namely for any $x, V_{x}(r)$ (the volume of the ball $B(x, r)$ ) is bounded from below by $\delta r^{2 m}$ for some positive constant $\delta$, in [N6] we proved that the Kähler-Ricci flow (2.1) has a long time solution with the initial data $g(x, 0)=g_{0}(x)$. Moreover, there exists a constant $A=A(M)>0$ such that $t \gg 1$,

$$
t \mathcal{R}(x, t) \leq A .
$$

Applying Theorem 6.1 to this situation we can show the following result.

Corollary 6.9. Let $\left(M, g_{0}\right)$ be a complete Kähler manifold as above. Let $g(x, t)$ be a long time solution to Kähler-Ricci flow as above. For any $\left(x_{j}, t_{j}\right)$ with $t_{j} \rightarrow \infty$ and $\frac{r_{0}^{2}\left(x_{j}, x_{0}\right)}{t_{j}} \leq C$ for some fixed point $x_{0} \in M$ and $C>0$ (where $r_{0}(x, y)$ is the distance function with respect to the initial metric $g(0))$, define $g_{j}(t)=\frac{1}{t_{j}} g\left(t_{j} t\right)$. Then the pointed sequence $\left(M, x_{j}, g_{j}(x, t)\right)$ sub-sequentially converges to a gradient expanding Kähler-Ricci soliton $\left(M_{\infty}, x_{\infty}, g_{\infty}(t)\right)$.

Proof. For any $t$, denote by $B_{t}(x, r)$ the ball of radius $r$ with respect to $g(t)$, and by $V_{t}(x, r)$ the volume of this ball (with respect to $g(t)$ ). By Theorem 2.2 of [NT3], we know that $V_{t}(x, r) \geq \delta r^{2 m}$. Together with (6.21) we have the injectivity radius bounded from below uniformly 
for $g_{j}(t)$. Therefore, by Hamilton's compactness theorem, $\left(M, x_{j}, g_{j}(t)\right)$ sub-sequentially converges to $\left(M_{\infty}, x_{\infty}, g_{\infty}(t)\right)$, a solution to KählerRicci flow defined on $M_{\infty} \times(0, \infty)$. The only thing we need to prove is that $\left(M_{\infty}, g_{\infty}(t)\right)$ is an expanding soliton. It is easy to see that $\left(M_{\infty}, g_{\infty}\right)$ also has bounded nonnegative bisectional curvature and the maximum volume growth. By Corollary 1 of [N6], we know that $M_{\infty}$ is topologically $\mathbb{R}^{2 m}$. The fact that it is an expanding soliton follows from the monotonicity consideration. Let us adapt the notations from Theorem 6.1. Let $\left(x_{0}, 0\right)$ be the fixed reference point, with respect to which we defined the forward reduced distance function $\ell_{+}(x, t)$, then the second forward reduced volume function $\tilde{u}(x, t)$ and the second forward reduced volume $\tilde{\theta}_{+}(t)$. By the proof of Theorem 6.1 we have that

$$
\frac{d}{d t} \tilde{\theta}_{+}(t)=-\int_{M} \frac{K}{t^{3 / 2}} \tilde{u} d \mu_{t}
$$

from which we can conclude that

$$
\tilde{\theta}_{+}\left(t_{j}\right)-\tilde{\theta}_{+}\left(2 t_{j}\right)=\int_{t_{j}}^{2 t_{j}} \frac{1}{t^{3 / 2}} \int_{M} K \tilde{u} d \mu_{t} d t
$$

Since $\tilde{\theta}_{+}\left(t_{j}\right)-\tilde{\theta}_{+}\left(2 t_{j}\right) \rightarrow 0$ as $j \rightarrow \infty$,

$$
\int_{t_{j}}^{2 t_{j}} \frac{1}{t^{3 / 2}} \int_{M} K \tilde{u} d \mu_{t} d t \rightarrow 0 .
$$

Taking limit we have that on $\left(M_{\infty}, g_{\infty}(t)\right)$

$$
\int_{1}^{2} \frac{1}{t^{3 / 2}} \int_{M_{\infty}} K \tilde{u}_{\infty} d \mu_{t} d t=0
$$

Here $\tilde{u}_{j}(y, t)=\frac{e^{-\ell_{+}\left(y, t_{j} t\right)}}{(\pi t)^{m}}$ and $\tilde{u}_{\infty}=\frac{e^{-\ell_{+}^{(\infty)}(y, t)}}{(\pi t)^{m}}$ with $\ell_{+}^{(\infty)}(y, t)$ being the limit of $\ell_{+}\left(y, t_{j} t\right)$. By the assumption on $x_{j}$, it is easy to check that $\ell\left(x_{j}, t_{j} t\right) \leq C$ for some $C$ independent of $j$, hence $\tilde{u}_{\infty}>0$ at least somewhere on $M_{\infty}$. (Strictly speaking one has to check that $\frac{1}{t_{j}^{1 / 2}} K\left(t_{j} t\right)$ stay bounded in finite balls centered at $x_{j}$ with respect to $g_{j}$, which can be verified by the derivative estimates of Shi.) The conclusion now follows from [C2] (see also Theorem 4.1 of [N2] for a short proof) since $K=0$ implies that the linear trace LYH quantity $H$ achieves its minimum (zero) somewhere.

q.e.d.

\section{Remark 6.10.}

(1) Corollary 6.9 simply says that the blow-down limit of a Type III solution is an expanding soliton. The result is in some sense dual to Proposition 11.2 of $[\mathbf{P}]$. It is also similar to the mean curvature flow result of $[\mathbf{H u}]$. The maximum volume growth assumption can be weakened to a certain $\kappa$-noncollapsing condition in terms of the lower bound of $\tilde{\theta}_{+}(t)$. 
(2) It has been proved in $[\mathbf{C T 1}$ ( see also [CT2] for more recent exciting progresses towards the uniformization problem) that a gradient expanding Kähler-Ricci soliton must be biholomorphic to $\mathbb{C}^{m}$.

(3) One can have a similar result for the Riemannian case, replacing the nonnegativity of the bisectional curvature by the nonnegativity of the curvature operator.

\section{Appendix: A parabolic relative volume comparison theorem}

Recall that Cheeger and Yau proved that on a complete Riemannian manifold with nonnegative Ricci curvature, the heat kernel $H(x, y, \tau)$ (the fundamental solution of the operator $\left(\frac{\partial}{\partial \tau}-\Delta\right)$ ) has the lower estimate

$$
H(x, y, \tau) \geq \frac{1}{(4 \pi \tau)^{\frac{n}{2}}} \exp \left(-\frac{r^{2}(x, y)}{4 \tau}\right)
$$

where $r(x, y)$ is the distant function on the manifold. This fact can be derived out of the maximum principle and the differential inequality

$$
\left(\frac{\partial}{\partial \tau}-\Delta\right)\left(\frac{1}{(4 \pi \tau)^{\frac{n}{2}}} \exp \left(-\frac{r^{2}(x, y)}{4 \tau}\right)\right) \leq 0 .
$$

Integrating on the manifold $M$, this differential inequality also implies the monotonicity (monotone non-increasing) of the integral

$$
\tilde{V}\left(x_{0}, \tau\right):=\int_{M} \frac{1}{(4 \pi \tau)^{\frac{n}{2}}} \exp \left(-\frac{r^{2}\left(x_{0}, y\right)}{4 \tau}\right) .
$$

In $[\mathbf{P}]$, Perelman discovered a striking analogue of this comparison result for the Ricci flow geometry. More precisely, he introduced a length function, called the reduced distance,

$$
\ell_{x_{0}, g(\tau)}(y, \bar{\tau}):=\inf _{\gamma} \frac{1}{2 \sqrt{\tau}} \int_{0}^{\bar{\tau}} \sqrt{\tau}\left(\left|\gamma^{\prime}(\tau)\right|^{2}+R(\gamma(\tau), \tau)\right) d \tau
$$

for all $\gamma(\tau)$ with $\gamma(0)=x_{0}, \gamma(\bar{\tau})=y$ (in the right context, we often omit the subscript $\left.x_{0}, g(\tau)\right)$, and a functional, called the reduced volume,

$$
\tilde{V}_{g(\tau)}\left(x_{0}, \tau\right):=\int_{M} \frac{1}{(4 \pi \tau)^{\frac{n}{2}}} \exp (-\ell(y, \tau))
$$

with respect to a solution $g_{i j}(x, \tau)$ to the backward Ricci flow

$$
\frac{\partial}{\partial \tau} g_{i j}=2 R_{i j}
$$

on $M \times[0, a]$. Perelman proved further that $\tilde{V}_{g(\tau)}\left(x_{0}, \tau\right)$ is monotone non-increasing in $\tau$. In fact, he showed this monotonicity result via a space-time relative comparison result and used it to give a more flexible 
proof of the $\kappa$-noncollapsing on the solution to Ricci flow, defined on $M \times[0, T]$, for any given finite time $T$ with given initial data.

In $[\mathbf{P}]$, Perelman also discovered a more rigid monotone quantity, the entropy functional. These two functionals can be related through a differential inequality of LYH type. One can refer to $[\mathbf{C L N}]$ for an exposition on this relation. The entropy is stronger but less flexible. The entropy monotonicity has its analogue for the positive solutions to a linear heat equation on a fixed Riemannian manifold with non-negative Ricci curvature. This was derived in [N3], where the author also observed that the entropy defined on a complete Riemannian manifold $M$ with non-negative Ricci curvature is related to the volume growth of the manifold. More precisely, let

$$
\mathcal{W}(f, \tau):=\int_{M}\left(\tau|\nabla f|^{2}+f-n\right) \frac{e^{-f}}{(4 \pi \tau)^{\frac{n}{2}}} d \mu
$$

for any $\tau>0$ and $C^{1}$ function $f$ with $\int_{M} \frac{e^{-f}}{(4 \pi \tau)^{\frac{n}{2}}} d \mu=1$. If $u(x, \tau)=$ $\frac{e^{-f}}{(4 \pi \tau)^{\frac{n}{2}}}$ is a solution to the heat equation, it was proved in [N3] that $\mathcal{W}(f, \tau)$ is monotone non-increasing in $\tau$. Moreover, it was shown that

$$
\lim _{\tau \rightarrow \infty} \mathcal{W}(f, \tau)=\log \left(\lim _{\tau \rightarrow \infty} \tilde{V}_{x_{0}}(\tau)\right)
$$

if $u(x, \tau)$ is a fundamental solution originated at $x_{0}$. In fact,

$$
\lim _{\tau \rightarrow \infty} \tilde{V}_{x_{0}}(\tau)=\lim _{r \rightarrow \infty} \frac{V_{x_{0}}(r)}{\omega_{n} r^{n}}
$$

where $V_{x_{0}}(r)$ is the volume of a ball of radius $r$, which is independent of $x_{0}$. (The limit on the right hand side above is also called the cone angle at infinity).

Motivated by this close connection between the Ricci flow geometry for a family of metrics and the Riemannian geometry of a fixed Riemannian metric, it is natural to seek a localized version of the above mentioned Cheeger-Yau's result on the monotonicity of the reduced volume $\tilde{V}_{x_{0}}(\tau)$. In fact, a local version of the heat kernel comparison was also carried out in the paper of $[\mathbf{C Y}]$ from a PDE point of view. However, Perelman's localization in the case of Ricci flow geometry is along the line of comparison geometry, and very much different from CheegerYau's localization by considering the Dirichlet or Neumann boundary value problem for the heat equation. This leads us to formulate a different relative (local) volume comparison theorem in this section, which is the linear analogue of Perelman's formulation for Ricci flow. It can be viewed as a parabolic version of the (classical) relative volume comparison theorem in the standard Riemannian geometry. The interested readers may want to compare these two results in more detail. The 
relation between the classical relative volume comparison theorem and the new one here is very similar to the one between the monotonicity formula for the minimal submanifolds in $\mathbb{R}^{n}$ and Huisken's monotonicity for mean curvature flow in $\mathbb{R}^{n}$ (as well as Ecker's localized version).

In order to state our result, let us first fix some notations. It is our hope that the exposition below is detailed enough to be helpful in understanding $[\mathbf{P}]$ better. Fix a point $x_{0} \in M$. Let $\gamma(\tau)(0 \leq \tau \leq \bar{\tau})$ be a curve parameterized by the time variable $\tau$ with $\gamma(0)=x_{0}$. Here we imagine that we have a time function $\tau$, with which some parabolic equation is associated. Define the $\mathcal{L}$-length by

$$
\mathcal{L}(\gamma)(\bar{\tau})=\int_{0}^{\bar{\tau}} \sqrt{\tau}\left|\gamma^{\prime}(\tau)\right|^{2} d \tau
$$

We can define the $\mathcal{L}$-geodesic to be the curve which is the critical point of $\mathcal{L}(\gamma)$. The simple computation shows that the first variation of $\mathcal{L}$ is given by

$$
\delta \mathcal{L}(\gamma)=2 \sqrt{\bar{\tau}}\langle Y, X\rangle(\bar{\tau})-2 \int_{0}^{\bar{\tau}} \sqrt{\tau}\left(\left\langle\nabla_{X} X+\frac{1}{2 \tau} X, Y\right\rangle\right) d \tau,
$$

where $Y$ is the variational vector field, from which one can write down the $\mathcal{L}$-geodesic equation. It is an easy matter to see that $\gamma$ is a $\mathcal{L}$-geodesic if and only if $\gamma(\sigma)$ with $\sigma=2 \sqrt{\tau}$ is a geodesic. In other words, a $\mathcal{L}$ geodesic is a geodesic after certain re-parametrization. Here we insist all curves are parameterized by the 'time'-variable $\tau$. One can check that for any $v \in T_{x_{0}} M$ there exists a $\mathcal{L}$-geodesic $\gamma(\tau)$ such $\left.\frac{d}{d \sigma}(\gamma(\sigma))\right|_{\sigma=0}=v$. Notice that the variable $\sigma$ scales in the same manner as the distance function on $M$. So it is more convenient to work with $\sigma$.

We then define the $\mathcal{L}$-exponential map by

$$
\mathcal{L} \exp _{v}(\bar{\sigma}):=\gamma_{v}(\bar{\sigma})
$$

if $\gamma_{v}(\sigma)$ is a $\mathcal{L}$-geodesic satisfying that

$$
\lim _{\sigma \rightarrow 0} \frac{d}{d \sigma}\left(\gamma_{v}(\sigma)\right)=v
$$

It is also illuminating to go one dimensional higher by considering the manifold $\widetilde{M}=M \times[0,2 \sqrt{T}]$ and the space-time exponential map $\widetilde{\exp }\left(\tilde{v}^{a}\right)=\left(\mathcal{L} \exp _{\frac{v^{a}}{a}}(a), a\right)$, where $\tilde{v}^{a}=\left(v^{a}, a\right)$. Denote $\frac{v^{a}}{a}$ simply by $v^{1}$ and $\left(v^{1}, 1\right)$ by $\tilde{v}^{1}$. Also let $\tilde{\gamma}_{\tilde{v}^{a}}(\eta)=\widetilde{\exp }\left(\eta \tilde{v}^{a}\right)$. It is easy to see that

$$
\tilde{\gamma}_{\tilde{v}^{a}}(\eta)=\tilde{\gamma}_{\tilde{v}^{1}}(\eta a) .
$$

This shows that

$$
\left.d \widetilde{\exp }\right|_{(0,0)}=\text { identity. }
$$

Computing the second variation of $\mathcal{L}(\gamma)$ gives that

$$
\delta^{2} \mathcal{L}(\gamma)=2 \sqrt{\bar{\tau}}\left\langle\nabla_{Y} Y, X\right\rangle+\int_{0}^{\bar{\tau}} 2 \sqrt{\tau}\left(\left|\nabla_{X} Y\right|^{2}-R(X, Y, X, Y)\right) d \tau
$$


where $Y$ is a given variational vector field. Let $(y, \bar{\sigma})=\left(\mathcal{L} \exp _{v}(\bar{\sigma}), \bar{\sigma}\right)$. Consider the variation which is generated by $(w, 0)$. Namely consider the family

$$
U(s, \sigma)=\left(\mathcal{L} \exp _{v+s w}(\sigma), \sigma\right)=\widetilde{\exp }((\sigma(v+s w), \sigma)) .
$$

Direct calculation shows that

$$
\frac{D U}{\partial \sigma}(0,0)=(v, 1), \quad \frac{D U}{\partial s}(0,0)=0
$$

and that the Jacobi field $\tilde{J}_{w}(\sigma)=\left(J_{w}(\sigma), 0\right)$ is given by

$$
\left.\frac{D U}{\partial s}\right|_{s=0}(\sigma)=d \widetilde{\exp }((\sigma w, 0))
$$

with the initial velocity

$$
\nabla_{\frac{\partial}{\partial \sigma}}\left(\left.\frac{D U}{\partial s}\right|_{s=0}\right)(0)=(w, 0) .
$$

(One can define the Jacobi operator to be the linear second order operator associated with the quadratic form in the right-hand side of (7.7). One calls a vector field along $\gamma$ a $\mathcal{L}$-Jacobi field if it satisfies the Jacobi equation. It is easy to show that the variational vector field of a family of $\mathcal{L}$-geodesics satisfies the Jacobi equation as in the standard Riemannian geometry. In fact, the $\mathcal{L}$-Jacobi field turns out to be just the regular Jacobi-field after re-parametrization.) This shows that

$$
(d \mathcal{L} \exp )_{w}(\sigma)=J_{w}(\sigma)
$$

and

$$
d \widetilde{\exp }((\sigma w, 0))=\tilde{J}_{w}(\sigma) .
$$

Now we can conclude that $(y, \sigma)$ is a regular value of the map $\widetilde{\exp }$ (and $y$ is a regular value of $\left.\mathcal{L} \exp _{(\cdot)}(\sigma)\right)$ if and only if that any Jacobi field $\tilde{J}$ with initial condition as above does not vanish at $\sigma$. We can introduce the concept of a conjugate point (with respect to $x_{0}$ ) as in the classical case. We can define the set

$$
D(\bar{\sigma}) \subseteq T_{x_{0}} M
$$

to be the collection of vectors $v$ such that $\left(\mathcal{L} \exp _{v}(\sigma), \sigma\right)$ is a $\mathcal{L}$-geodesic along which there is no conjugate point up to $\bar{\sigma}$. Similarly we can define the set

$$
C(\bar{\sigma}) \subseteq T_{x_{0}} M
$$

to be the collection of vectors $v$ such that $\left(\mathcal{L}_{v}(\sigma), \sigma\right)$ is a minimizing $\mathcal{L}$-geodesic up to $\bar{\sigma}$. One can see easily that $D(\sigma)$ and $C(\sigma)$ decrease (as sets) as $\sigma$ increases. For any measurable subset $A \subseteq T_{x_{0}} M$ we can define

$$
D_{A}(\sigma)=A \cap D(\sigma) \text { and } \quad C_{A}(\sigma)=A \cap C(\sigma) .
$$


Following $[\mathbf{P}]$ we also introduce the $\ell$-'distance' function.

$$
\left.\ell_{x_{0}}(y, \bar{\tau})=\frac{1}{2 \sqrt{\bar{\tau}}} L_{x_{0}}(y, \bar{\tau}), \quad \text { where } L_{x_{0}}(y, \bar{\tau})=\inf _{\gamma} \mathcal{L}(\gamma)\right) .
$$

Here our $\ell$ is defined for a fixed background metric. We also omit the subscript $x_{0}$ in the context where the meaning is clear. The very same consideration as in $[\mathbf{P}]$, as well as in the standard Riemannian geometry, shows that

$$
\begin{aligned}
& |\nabla \ell|^{2}=\frac{1}{\bar{\tau}} \ell \\
& \ell_{\tau}=-\frac{1}{\bar{\tau}} \ell
\end{aligned}
$$

and

$$
\Delta \ell \leq \frac{n}{2 \bar{\tau}}-\frac{1}{\bar{\tau}^{\frac{3}{2}}} \int_{0}^{\bar{\tau}} \tau^{\frac{3}{2}} \operatorname{Ric}(X, X) d \tau
$$

where $X=\gamma^{\prime}(\tau)$ with $\gamma(\tau), 0 \leq \tau \leq \bar{\tau}$ being the minimizing $\mathcal{L}$-geodesic joining $x_{0}$ to $y$. Putting (7.10)-(7.12) together, one obtains a new proof of the result of Cheeger-Yau, which asserts that if $M$ has nonnegative Ricci curvature, then

$$
\left(\frac{\partial}{\partial \tau}-\Delta\right)\left(\frac{e^{-\ell(y, \tau)}}{(4 \pi \tau)^{\frac{n}{2}}}\right) \leq 0 .
$$

Namely $\frac{e^{-\ell(y, \tau)}}{(4 \pi \tau)^{\frac{n}{2}}}$ is a sub-solution of the heat equation. In particular,

$$
\frac{d}{d \tau} \int_{M} \frac{e^{-\ell(y, \tau)}}{(4 \pi \tau)^{\frac{n}{2}}} d \mu \leq 0 .
$$

Using the above geometric consideration, one can think of the above result of Cheeger-Yau as a parabolic volume comparison with the respect to the positive measure $\frac{e^{-\ell(y, \tau)}}{(4 \pi \tau)^{\frac{n}{2}}} d \mu$. Recall that the well-known Bishop-Gromov volume comparison states that if $M$ has nonnegative Ricci curvature

$$
\frac{d}{d r}\left(\frac{1}{r^{n-1}} \int_{S_{x_{0}}(r)} d A\right) \leq 0
$$

where $S_{x_{0}}(r)$ denotes the boundary of the geodesic ball centered at $x_{0}$ with radius $r, d A$ is the induced area measure. The by-now standard relative volume comparison can be formulated in a way similar to the above. Let $A$ be a measurable subset of $S^{n-1} \subset T_{x_{0}} M$. One can define $C_{A}(r)$ to be the collection of vectors $r v$ with $v \in A$ such that the geodesic $\exp _{x_{0}}(s v)$ is minimizing for $s \leq r$, where $\exp _{x_{0}}(\cdot)$ is the (classical) exponential map. Then the classical relative volume comparison 
theorem (cf. $[\mathbf{G r}]$ ) asserts that if $M$ has nonnegative Ricci curvature,

$$
\frac{d}{d r}\left(\frac{1}{r^{n-1}} \int_{\exp \left(C_{A}(r)\right)} d A\right) \leq 0 .
$$

The following is a parabolic version of such a relative volume comparison theorem parallel to Perelman's work on Ricci flow geometry.

Theorem 7.1. Assume that $M$ has nonnegative Ricci curvature. Then

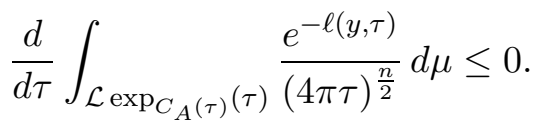

Proof. The proof follows a similar argument as in $[\mathbf{P}]$. Using the notation in the above discussion, first we observe that

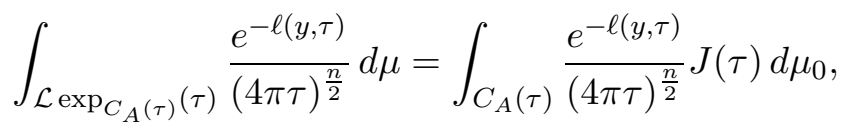

where $J(\tau)$ is the Jacobian of $\mathcal{L} \exp _{(\cdot)}(\tau)$. Since $C_{A}(\tau)$ is decreasing in $\tau$, it suffices to show that

$$
\frac{d}{d \tau}\left(\frac{e^{-\ell(y, \tau)}}{(4 \pi \tau)^{\frac{n}{2}}} J(\tau)\right) \leq 0 .
$$

This follows from (7.10)-(7.11), the fact that

$$
\frac{d}{d \tau} e^{-\ell}=\left(-\frac{\ell}{\bar{\tau}}+\langle\nabla \ell, X\rangle(\bar{\tau})\right) e^{-\ell}=0
$$

(since $\nabla \ell(\bar{\tau})=X)$, and the claim that

$$
\frac{d}{d \tau} \log J(\bar{\tau}) \leq \frac{n}{2 \bar{\tau}}-\frac{1}{\bar{\tau}} \int_{0}^{\bar{\tau}} \tau^{\frac{3}{2}} \operatorname{Ric}(X, X) d \tau .
$$

The estimate (7.15) follows exactly the same proof as in $[\mathbf{P}]$, via the second variation formula (7.7) and its consequence on the Hessian comparison:

$$
\operatorname{Hess}(L)(\bar{Y}, \bar{Y}) \leq \int_{0}^{\bar{\tau}} 2 \sqrt{\tau}\left(\left|\nabla_{X} Y\right|^{2}-R(X, Y, X, Y)\right) d \tau
$$

where $Y(\tau)$ is a vector field along the minimizing $\mathcal{L}$-geodesic $\gamma(\tau)$ joining $x_{0}$ to $y$ with $\gamma(\bar{\tau})=y$, satisfying $Y(\bar{\tau})=\bar{Y}$ and

$$
\nabla_{X} Y-\frac{1}{2 \tau} Y=0 .
$$


Better comparison between Theorem 7.1 and the classical relative volume comparison can be seen by replacing $\widetilde{M}$ with $M, M \times\{a\}$ with $S_{x_{0}}(a)$. Notice that in $[\mathbf{P}]$, one does need such a result (not just the global version on the monotonicity of $\left.\tilde{V}_{g(\tau)}\left(x_{0}, \tau\right)\right)$ to prove the noncollapsing result on finite time solution to Ricci flow. This and the potential application to the study of Riemannian geometry of our linear version, justify the spelling out of this result in spite of its simplicity.

Remark 7.2. One can formulate a similar parabolic version of the volume comparison result for the case $\operatorname{Ric}(M) \geq-(n-1) K$. We leave that to the interested readers.

In [N3], we showed that the lower bound on the entropy $\inf _{0 \leq \tau \leq T} \mu(\tau)$ (please see [N3] for definition) implies the non-collapsing of volume of a ball of radius $r$ for $r^{2} \leq T$. This corresponds (but is considerably easier than) to the $\kappa$-non-collapsing result of Perelman on the solutions to Ricci flow, via the monotonicity of entropy functional. Using Theorem 7.1, one can have the following consequence, which is just the linear version of Perelman's second proof on the $\kappa$-non-collapsing result.

Lemma 7.3. Let $M$ be a complete Riemannian manifold with nonnegative Ricci curvature. Assume that $B\left(x_{0}, r\right)$ is $\kappa$-collapsed in the sense that

$$
V\left(x_{0}, r\right) \leq \kappa r^{n}
$$

Then there exists $C=C(n)>0$ such that

$$
\tilde{V}\left(\kappa^{\frac{1}{n}} r^{2}\right) \leq C\left(\sqrt{\kappa}+\exp \left(-\frac{1}{8 \kappa^{\frac{1}{n}}}\right)\right)
$$

Namely, the smallness of the relative volume $\frac{V\left(x_{0}, r\right)}{r^{n}}$ is equivalent to the smallness of the 'reduced volume' $\tilde{V}\left(r^{2}\right)$.

Notice that the lemma can also be proved by direct computations and the Bishop volume comparison theorem, without using Theorem 7.1 (cf. [CLN] for details).

Finally we present here a second variation formula for Perelman's $\mathcal{L}$ length, which is slightly different from the original one stated in $[\mathbf{P}]$. The hope is that this new form will be easier to use. Recall that for the backward Ricci flow $\frac{\partial}{\partial \tau} g_{i j}=2 R_{i j}$ on $M \times[0, T]$, for any path $\gamma(\tau)$ joining $\left(x_{0}, 0\right)$ to $(y, \bar{\tau})$, Perelman defined the $\mathcal{L}$-length

$$
\mathcal{L}(\gamma)=\int_{0}^{\bar{\tau}} \sqrt{\tau}\left(\left|\gamma^{\prime}\right|^{2}+\mathcal{R}\right) d \tau
$$


The first variation computation gives that for any variational vector field $Y$,

$$
\begin{aligned}
\delta_{Y}(\mathcal{L}(\gamma))= & \left.2(\sqrt{\tau}\langle Y, X\rangle)\right|_{0} ^{\bar{\tau}} \\
& -2 \int_{0}^{\bar{\tau}} \sqrt{\tau}\left\langle\nabla_{X} X-\frac{1}{2} \nabla \mathcal{R}+2 R c(X)+\frac{1}{2 \tau} X, Y\right\rangle d \tau .
\end{aligned}
$$

Here $X=\gamma^{\prime}$ and $R c$ is the $(1,1)$ tensor obtained from the Ricci tensor by lifting.

Let $\gamma(\tau, y, w)$ be variation of $\gamma(\tau)$ of two parameters with the variational vector fields $Y$ and $W$. The second variation computation gives

$$
\begin{aligned}
\delta_{Y, W}^{2}(\mathcal{L}(\gamma))= & {\left.\left[2 \sqrt{\tau}\left(\left\langle\nabla_{W} Y, X\right\rangle+\left\langle Y, \nabla_{X} W\right\rangle\right)\right]\right|_{0} ^{\bar{\tau}}-2 \int_{0}^{\bar{\tau}} \sqrt{\tau}\langle\mathcal{J}(W), Y\rangle } \\
& +\left\langle\nabla_{X} X-\frac{1}{2} \nabla \mathcal{R}+2 R c(X)+\frac{1}{2 \tau} X, \nabla_{W} Y\right\rangle d \tau .
\end{aligned}
$$

Here $\mathcal{J}(W)$ is the Jacobi operator given by

$$
\begin{aligned}
\mathcal{J}(W)= & \nabla_{X} \nabla_{X} W+R(X, W) X-\frac{1}{2} \nabla_{W} \nabla \mathcal{R}+2\left(\nabla_{W} R c\right)(X) \\
& +2 R c\left(\nabla_{X} W\right)+\frac{1}{2 \tau} \nabla_{X} W .
\end{aligned}
$$

In the case $\gamma$ is a $\mathcal{L}$-geodesic the last term in the second variation formula vanishes. We then have

$$
\begin{aligned}
\delta_{Y, W}^{2}(\mathcal{L}(\gamma))= & {\left.\left[2 \sqrt{\tau}\left(\left\langle\nabla_{W} Y, X\right\rangle+\left\langle Y, \nabla_{X} W\right\rangle\right)\right]\right|_{0} ^{\bar{\tau}} } \\
& -2 \int_{0}^{\bar{\tau}} \sqrt{\tau}\langle\mathcal{J}(W), Y\rangle d \tau .
\end{aligned}
$$

It is desirable to write $\mathcal{J}(W)$ in a better form. To do that we define a operator $D_{\tau}$ on any vector $Y$ as $D_{\tau} Y=\frac{d}{d \tau} Y+R c(Y)$. Direct computation shows that

$$
\mathcal{J}(W)=D_{\tau} D_{\tau} W+\frac{1}{2 \tau} D_{\tau} W+\widetilde{\mathcal{R} m}(W)
$$

where

$$
\begin{aligned}
\widetilde{\mathcal{R} m}(W)= & -\frac{\partial R c}{\partial \tau}(W)-\frac{1}{2} \nabla_{W} \nabla \mathcal{R}-R c(R c(W))+R(X, W) X \\
& -\frac{1}{2 \tau} R c(W)+2\left(\nabla_{W} R c\right)(X)-2\left(\nabla_{X} R c\right)(W) \\
& +(\langle(\nabla \cdot R c)(X), W\rangle)^{*}-\left(\nabla_{W} R c\right)(X)
\end{aligned}
$$

Here $(\langle(\nabla . R c)(X), W\rangle)^{*}$ is the dual vector of the one form $\langle(\nabla \cdot R c)(X), W\rangle$. It is easy to check that $\widetilde{\mathcal{R} m}(W)$ is self-adjoint and more importantly, $\langle\widetilde{\mathcal{R} m}(W), W\rangle$ is nothing but Hamilton's differential 
Harnack expression for the shrinkers, which can be viewed as a spacetime curvature by $[\mathbf{C C 1}]$. By integration by parts we have that

$$
\begin{aligned}
\delta_{Y, W}^{2}(\mathcal{L}(\gamma))= & {\left.\left[2 \sqrt{\tau}\left(\left\langle\nabla_{W} Y, X\right\rangle-\langle R c(Y), W\rangle\right)\right]\right|_{0} ^{\bar{\tau}} } \\
& +2 \int_{0}^{\bar{\tau}} \sqrt{\tau}\left(\left\langle D_{\tau} W, D_{\tau} Y\right\rangle-\langle\widetilde{\mathcal{R} m}(W), Y\rangle\right) d \tau .
\end{aligned}
$$

The last integration above can be called the index form, whose EulerLagrange equation is just $\mathcal{J}(W)=0$. Using the easily-proved fact that the Jacobi field minimizes the index form if there is no conjugate point along $\gamma$, one can recover the key estimate (7.9) of $[\mathbf{P}]$ (see also $[\mathbf{T o}]$ ). Similar consideration can be applied to the steady reduced distance (an expression suggested to us by Ilmanen). Define

$$
\mathcal{L}^{0}(\gamma)=\int_{0}^{\bar{\tau}}\left(\left|\gamma^{\prime}\right|^{2}+\mathcal{R}\right) d \tau
$$

If $\gamma$ is a $\mathcal{L}^{0}$-geodesic we have that

$$
\begin{aligned}
\delta_{Y, W}^{2}\left(\mathcal{L}^{0}(\gamma)\right)= & \left.2\left(\left\langle\nabla_{W} Y, X\right\rangle-\langle R c(Y), W\rangle\right)\right|_{0} ^{\bar{\tau}} \\
& +2 \int_{0}^{\bar{\tau}}\left(\left\langle D_{\tau} W, D_{\tau} Y\right\rangle-\left\langle\widetilde{\mathcal{R} m}^{0}(W), Y\right\rangle\right) d \tau
\end{aligned}
$$

where $\widetilde{\mathcal{R} m}^{0}(W)=\widetilde{\mathcal{R} m}(W)+\frac{1}{2 \tau} R c(W)$, which is Hamilton's matrix Harnack expression for steady solitons.

\section{References}

[A] B. Andrews, Harnack inequalities for evolving hypersurfaces, Math. Z. 217 (1994) 179-197, MR 1296393, Zbl 0807.53044.

[B] S. Bando, On classification of three-dimensional compact Kähler manifolds of nonnegative bisectional curvature, J. Differential Geom. 19 (1984) 283-297, MR 0755227, Zbl 0547.53034.

[C1] H.-D. Cao, On Harnack inequalities for the Kähler-Ricci flow, Invent. Math. 109 (1992) 247-263, MR 1172691, Zbl 0779.53043.

[C2] , Limits of solutions to the Kähler-Ricci flow, J. Differential Geom. 45 (1997) 257-272, MR 1449972, Zbl 0889.58067.

[CN] H.-D. Cao \& L. Ni, Matrix Li-Yau-Hamilton estimates for heat equation on Kähler manifolds, Math. Ann. 331 (2005) 795-807, MR 2148797.

[CT1] A. Chau \& L.-F. Tam, A note on the uniformization of gradient Kähler-Ricci solitons, Math. Res. Lett. 12(1) (2005) 19-21, MR 2122726.

[CT2] , On the complex structure of noncompact Kähler manifolds with nonnegative bisectional curvature, preprint.

[CLY1] S.-Y. Cheng, P. Li, \& S.-T. Yau, On the upper estimate of the heat kernel of a complete Riemannian manifold, Amer. J. Math. 103(5) (1981) 1021-1063, MR 0630777, Zbl 0484.53035.

[CLY2] - Heat equations on minimal submanifolds and their applications, Amer. J. Math. 106(5) (1984) 1033-1065, MR 0761578, Zbl 0575.53038. 
[CY] J. Cheeger \& S.-T. Yau, A lower bound for the heat kernel, Comm. Pure Appl. Math. 34(4) (1981) 465-480, MR 0615626, Zbl 0481.35003.

[Ch] B. Chow, Interpolating between Li-Yau's and Hamilton's Harnack inequalities on a surface, J. Partial Differential Equations 11(2) (1998) 137-140, MR 1626999, Zbl 0943.58017.

[CC1] B. Chow \& S.-C. Chu, A geometric interpretation of Hamilton's Harnack inequality for the Ricci flow, Math. Res. Lett. 2(6) (1995) 701-718, MR 1362964, Zbl 0856.53030.

[CC2] , A geometric approach to the linear trace Harnack inequality for the Ricci flow, Math. Res. Lett. 3(4) (1996) 549-568, MR 1406020, Zbl 0868.58082

[CLN] B. Chow, P. Lu, \& L. Ni, Hamilton's Ricci Flow, in preparation.

$[\mathrm{CH}] \quad$ B. Chow \& R. Hamilton, Constrained and linear Harnack inqualities for parabolic equations, Invent. Math. 129 (1997) 213-238, MR 1465325.

[CK1] B. Chow \& D. Knopf, New Li-Yau-Hamilton inequalities for the Ricci flow via the space-time approach, J. Differential Geom. 60(1) (2002) 1-54, MR 1924591, Zbl 1048.53026.

[CK2] , The Ricci Flow, vol. I: an introduction, Math. Surveys and Monographs, AMS, 110, 2004, MR 2061425.

[CS] M. Cornalba \& B. Shiffman, A counterexample to the 'Transcendental Bézout problem', Ann. of Math. 96(2) (1972) 402-406, MR 0311937.

[E1] K. Ecker, A local monotonicity formula for mean curvature flow, Ann. of Math. (2) 154(2) (2001) 503-525, MR 1865979, Zbl 1007.53050.

[E2] Regularity Theory for Mean Curvature Flow, Progress in Nonlinear Differential Equations and their Applications, 57, Birkhuser Boston, Inc., Boston, MA, 2004, MR 2024995, Zbl 1058.53054.

[Ev1] L. Evans, A survey of entropy methods for partial differential equations, Bull. AMS. 41(4) (2004) 409-438, MR 2083636, Zbl 1053.35004.

[Ev2] Entropy and Partial Differential Equations, Lecture Notes at UC Berkeley.

[FIN] M. Feldman, T. Ilmanen, \& L. Ni, Entropy and reduced distance for Ricci expanders, Jour. Geom. Anal. 15 (2005) 49-62, MR 2132265, Zbl 1071.53040.

[G] P. Griffiths, Entire Holomorphic Mappings in One and Several Complex Variables, Annals of Mathematics Studies, 85, Princeton Press, 1976, MR 0447638.

[GK] P. Griffiths \& J. King, Nevanlinna theory and holomorphic mappings between algebraic varieties, Acta. Math. 130 (1973) 145 -220, MR 0427690, Zbl 0258.32009.

[Gr] M. Gromov, Metric Structure for Riemannian and Non-Riemannian Spaces, Progress in Mathematics 152, Birkhuser Boston, Inc., Boston, MA, 1999, MR 1699320.

[Gu] C. Guenther, The fundamental solution on manifolds with time-dependent metrics, Jour. Geom. Anal. 12 (3) (2002) 425-436, MR 1901749, Zbl 1029.58018 .

[H1] R. Hamilton, The Harnack estimate for the Ricci flow, J. Differential Geom. 37(1) (1993) 225-243, MR 1316556, Zbl 0804.53023. 
[H2] A matrix Harnack estimate for the heat equation, Comm. Anal. Geom. 1(1) (1993) 113-126, MR 1230276, Zbl 0799.53048.

[H3] , Monotonicity formulas for parabolic flows on manifolds, Comm. Anal. Geom. 1(1) (1993) 127-137, MR 1230277, Zbl 0779.58037.

[Ho] L. Hörmander, An Introduction to Complex Analysis in Several Variables, 3rd Edition, North Holland, 1990, MR 1045639.

[Hu] G. Huisken, Asymptotic behavior for singularities of the mean curvature flow, J. Differential Geom. 31 (1990) 285-299, MR 1030675, Zbl 0694.53005.

[KM] K. Kodaira \& J. Morrow, Complex Manifolds, Holt, Rinehart and Winston, Inc. 1971, MR 0302937.

[LS] P. Li \& R. Schoen, $L^{p}$ and mean value properties of subharmonic functions on Riemannian manifolds, Acta Math. 153 (1984) 279-301, MR 0766266, Zbl 0556.31005.

[LT] P. Li \& G. Tian, On the heat kernel of the Bergmann metric on algebraic varieties, J. Amer. Math. Soc. 8(4) (1995) 857-877, MR 1320155, Zbl 0864.58058.

[LW] P. Li \& J. Wang, Comparison theorem for Kähler manifolds and positivity of spectrum, preprint.

[LY] P. Li \& S.-T. Yau, On the parabolic kernel of the Schrödinger operator, Acta Math. 156(3-4) (1986) 153-201, MR 0834612, Zbl 0611.58045.

[M1] N. Mok, An embedding theorem of complete Kähler manifolds of positive bisectional curvature onto affine algebraic varieties, Bull. Soc. Math. France 112(2) (1984) 197-250, MR 0788968, Zbl 0536.53062.

[M2] , The uniformization theorem for compact Kähler manifolds of nonnegative holomorphic bisectional curvature, J. Differential Geom. 27 (1988) 179-214, MR 0925119, Zbl 0642.53071.

[Mo] J. Moser, A Harnack inequality for parabolic differential equations, Comm. Pure Appl. Math. 17 (1964) 101-134, MR 0159139, Zbl 0149.06902.

[N1] L. Ni, The Poisson equation and Hermitian-Einstein metrics on complete Kähler manifolds, Indiana Univ. Math. J.51 (2002) 679-704, MR 1911050.

[N2] Monotonicity and Kähler-Ricci flow, Contemp. Math. 367 (2005) 149-165, MR 2115758.

[N3] _ The entropy formula for linear heat equation, Jour. Geom. Anal. 14 (2004), 85-98, MR 2030576, Zbl 1062.58028; addenda, Jour. Geom. Anal. 14 (2004) 329-334, MR 2051693.

[N4] , A monotonicity formula on complete Kähler manifold with nonnegative bisectional curvature, J. Amer. Math. Soc. 17 (2004) 909-946, MR 2083471.

[N5] - Ricci flow and nonnegativity of sectional curvature, Math. Res. Lett. 11 (2004) 883-904, MR 2106247.

[N6] _ Ancient solutions to Kähler-Ricci flow, Math. Res. Lett. 12 (2005) 633-654.

[NT1] L. Ni \& L.-F. Tam, Plurisubharmonic functions and the Kähler-Ricci flow, Amer. J. Math. 125 (2003), 623-654, MR 1981036, Zbl 1027.53076.

[NT2] , Plurisubharmonic functions and the structure of complete Kähler manifolds with nonnegative curvature, J. Differential Geom. 64 (2003) 457524, MR 2032112. 
[NT3] , Kähler-Ricci flow and the Poincaré-Lelong equation, Comm. Anal. Geom. 12 (2004) 111-141, MR 2074873, Zbl 1067.53054.

[P] G. Perelman, The entropy formula for the Ricci flow and its geometric applications, arXiv: math.DG/0211159.

[Ru] H. Rutishauser, Über Folgen und Scharen von analytischen und meromorphen Funktionen mehrerer Variabeln, sowie von analytischen Abbildungen, Acta Math. 83 (1950) 249-325, MR 0036322.

[Sh1] W.X. Shi, Deforming the metric on complete Riemannian manifolds, J. Differential Geom. 30 (1989), 223-301, MR 1001277, Zbl 0676.53044.

[Sh2] Ricci flow and the uniformization on complete noncompact Kähler manifolds, J. Differential Geom. 45 (1997), 94-220, MR 1443333, Zbl 0954.53043.

[St] W. Stoll, The growth of the area of a transcendental analytic set, I and II, Math. Ann. 156 (1964) 47-78, 144-170, MR 0166393, Zbl 0126.09502.

[To] M. Toda, Notes on noncollapsing theorems, notes on Perelman's paper.

[W1] H. Wu, The equidistribution Theory of Holomorphic Curves, Annals of Math. Studies, 64, Princeton University Press, 1970, MR 0273070.

[W2] _ Polynomial functions on complete Kähler manifolds, Several complex variables and complex geometry, Proc. Symp. Pure Math. 52 (1989) 601-610, MR 1128575, Zbl 0744.32003.

[Ye] R. Ye, Notes on reduced volume and asymptotic Ricci solitons of $\kappa$-solutions, http://www.math.lsa.umich.edu/research/ricciflow/perelman.html.

Department of Mathematics

University of California at San Diego

LA Jolla, CA 92093

E-mail address: lni@math.ucsd.edu 